\title{
Robust Task Space Finite-Time Chattering-Free Control of Robotic Manipulators
}

\author{
Mirosław Galicki
}

Received: 15 December 2015 / Accepted: 1 May 2016 / Published online: 12 July 2016

(C) The Author(s) 2016. This article is published with open access at Springerlink.com

\begin{abstract}
This work deals with the problem of the accurate task space control subject to finite-time convergence. Kinematic and dynamic equations of a rigid robotic manipulator are assumed to be uncertain. Moreover, unbounded disturbances, i.e., such structures of the modelling functions that are generally not bounded by construction, are allowed to act on the manipulator when tracking the trajectory by the end-effector. Based on suitably defined task space non-singular terminal sliding vector variable and the Lyapunov stability theory, we derive a class of absolutely continuous (chattering-free) robust controllers based on the estimation of a Jacobian transpose matrix, which seem to be effective in counteracting uncertain both kinematics and dynamics, unbounded disturbances and (possible) kinematic and/or algorithmic singularities met on the robot trajectory. The numerical simulations carried out for a 2DOF robotic manipulator with two revolute kinematic pairs and operating in a two-dimensional task space, illustrate performance of the proposed controllers.
\end{abstract}

Keywords Robotic manipulator - Task space trajectory tracking $\cdot$ Finite-time control $\cdot$ Lyapunov stability

M. Galicki ( $\square)$

Faculty of Mechanical Engineering, University of Zielona

Góra, Zielona Góra, Poland

e-mail: m.galicki@ibem.uz.zgora.pl

\section{Introduction}

Robotic manipulators have found an increasing interest in recent years in industry to useful practical tasks such as inserting a shaft into a bearing hole or an assembly of electronic components onto the small surface of printed circuit boards. These tasks require, by their nature, extremely high precision and stability of the performance. In most situations met in practice, the aforementioned tasks are specified in terms of a time parameterized geometric path (a trajectory expressed in Cartesian coordinates) to be tracked by the end-effector of either non-redundant or redundant manipulator.

In order to apply well known joint space control techniques (see e.g. works [1-3, 5, 14, 15, 72]) for tracking such a trajectory, an inverse or pseudoinverse kinematics algorithm has to be utilised to transform Cartesian coordinates into the corresponding joint coordinates. The process of kinematic inversion is both time consuming (there does not exist, in general, an analytic form of inverse mapping) and becomes very complicated when the Cartesian trajectory generates kinematic and/or algorithmic singularities [51]. Moreover such inverse mapping does not even exist, in general, for redundant manipulators. Thus, a controller to be designed for kinematically non-redundant and/or redundant robotic manipulators should accurately track desired end-effector trajectory expressed in task coordinates despite possible singularities met on this trajectory, uncertain both 
kinematic and dynamic equations, unknown payload to be transferred by the end-effector and external disturbances. Moreover, such controller has to generate at least absolutely continuous control signals (torques) to avoid undesirable chattering [7]. Due to the challenging nature of the aforementioned control design problems, many researchers have proposed different types of controllers. In such a context, one can distinguish three major approaches of controlling the non-redundant and/or redundant robotic manipulators in the task space. The control techniques offered in the first approach [16-25, 43-45] require the full knowledge of the dynamics neglecting the external disturbances. In the second approach, works [26-37] present adaptive control algorithms to compensate for parametric uncertainties in dynamic model including only the linearly parametrizable friction terms (viscous friction) and also neglecting the external (nonlinearly parametrizable) disturbances. Moreover, control laws from [26-36] use inverse or pseudo-inverse of either the exact or approximate Jacobian matrices. Recent study [37] estimates the pseudo-inverse by some non-singular matrix which is adaptively computed. Work [47] presents an adaptive scheme for the motion control of robotic manipulator subject to parametric uncertainties and globally bounded disturbances. In the absence of disturbances and under some sufficient conditions on gain parameters, control law from [47] is shown to ensure only globally ultimately bounded Cartesian tracking error. In the third approach, model based robust control schemes were proposed in works [38-40]. The controllers from $[38,39]$ ensure uniformly ultimately bounded endeffector and sub-task tracking despite the parametric uncertainties associated with the dynamic equations, an upper bound on the parameter accuracy and globally bounded external disturbances. A neural network based adaptive asymptotically stable control scheme, in the presence of model uncertainties and globally bounded external disturbances, has been designed in [40]. Using extended Jacobian, nominal values of the parameters of dynamic equations and the momentum feed-back disturbance observer, a trajectory tracking control law has been proposed in [46] without its stability analysis.

From the literature survey, it follows that all the aforementioned control schemes require either the knowledge of the nominal robot dynamic equations whose construction may not be a trivial task or involve all the adaptive terms multiplied by the regression matrix (neglecting the external nonlinearly parametrizable disturbances) that seems to be both complex to implement and very time consuming. Moreover, all the trajectory tracking algorithms (except of [37]) require explicit inverse or pseudoinverse of a Jacobian matrix, what may result in numerical instabilities due to (possible) kinematic and/or algorithmic singularities [51] met on the robot trajectory. In order to tackle the singular configurations, the use of damped least-squares has been proposed in works [41, 42] in lieu of the pseudoinverses. Nevertheless, this technique suffers from the tracking errors due to a long-term numerical integration drift. Furthermore, all the control schemes assume globally bounded disturbances when tracking the trajectory whereas e.g. a viscous friction term is globally unbounded. The assumption of global boundedness of external disturbances may lead to deterioration of the trajectory tracking accuracy. Finally, all the aforementioned controllers provide only at most asymptotic stability what may be insufficient for accomplishment of tasks requiring the extremely high precision (e.g. assembly of electronic components on the small surface of printed circuit boards). Consequently, all those algorithms are not able to generate continuous (chattering-free) controls resulting in finite-time stability of the equilibrium when (possible) singular configurations may appear on the trajectory, kinematic and dynamic equations are uncertain and (unbounded) disturbances act on the robotic manipulator.

This paper presents a significant generalisation of the results previously published in works $[15,70]$. Namely, work [15] solves the finite-time control problem for uncertain robot equations and desired (reference) trajectories expressed only in the generalised (joint) coordinates, neglecting the kinematic equations of the manipulator. On the other hand, work [70] introduces a class of controllers being finitetime stable in the task (Cartesian) space provided that dynamic equations are uncertain and kinematic equations of the manipulator are fully known. In the present study, a new task space non-singular terminal sliding manifold (TSM) and a kind of dynamic computed torque method are introduced to track the endeffector trajectory expressed in the task coordinates, subject to uncertain both kinematic and dynamic equations of the manipulator. The proposed secondorder non-linear TSM manifold makes it possible to 
simultaneously join the first order sliding mode approach possessing the finite-time control capabilities with the second order sliding mode techniques generating the (absolutely) continuous (chatteringfree) controls. The offered TSM consists of the task error acceleration part which is responsible for chattering elimination and an integral non-linear term containing both task error and its time-derivative which is responsible for the finite-time convergence. Consequently, the proposed TSM needs the knowledge of acceleration. It is worth to emphasise that, the task space finite-time control of robotic manipulators subject to uncertain both kinematic and dynamic equations, absolute continuity control (chattering-free) requirement and globally unbounded disturbances, is still a non-trivial problem whose solution is based in this work on introducing a new dynamic version of the static computed torque approach presented e.g. in works [8, 19]. By fulfilment of reasonable assumption regarding the estimation of the Jacobian matrix, the proposed control scheme is shown to be finite-time stable. The remainder of the paper is organised as follows. Section 2 formulates the finite-time trajectory tracking task. Section 3 sets up a class of task space robust absolutely continuous controllers based on the estimation of Jacobian matrix and solving the trajectory tracking problem in a finite-time subject to uncertain both kinematic and dynamic robot equations and unbounded disturbances. Section 4 presents computer simulations of the end-effector trajectory tracking by a 2DOF robotic manipulator, consisting of two revolute kinematic pairs and operating in two-dimensional task space. Finally, some concluding remarks are drawn in Section 5.

\section{Problem Formulation}

The robust control scheme designed in the next section is applicable to holonomic mechanical systems comprising both non-redundant and redundant robotic manipulators considered here which are described, in general, by the following dynamic equations, expressed in generalized (joint) coordinates $q=$ $\left(q_{1}, \ldots, q_{n}\right)^{T}[8]$ :

$M(q) \ddot{q}+H(q, \dot{q})+G(q)+D(t, q, \dot{q})=v$,

where $\dot{q}$ and $\ddot{q}$ represent the velocity and acceleration, respectively. The $n \times n$ inertia matrix $M(q)$ is positive definite and symmetric. $H=B(q)(\dot{q} \cdot \dot{q})+$ $C(q)\left(\dot{q}^{2}\right)$, where $B$ and $C$ are the $n \times \frac{n(n-1)}{2}$ and $n \times n$ matrices of coefficients of the Coriolis and centrifugal forces, respectively. $(\dot{q} \cdot \dot{q})$ and $\left(\dot{q}^{2}\right)$ are the symbolic notations for the $\frac{n(n-1)}{2}$-dimensional and $n$ dimensional vectors $(\dot{q} \cdot \dot{q})=\left(\dot{q}_{1} \dot{q}_{2}, \ldots, \dot{q}_{n-1} \dot{q}_{n}\right)^{T}$ and $\left(\dot{q}^{2}\right)=\left(\dot{q}_{1}^{2}, \ldots, \dot{q}_{n}^{2}\right)^{T}$, respectively. $v=$ $\left(v_{1}, \ldots, v_{n}\right)^{T}$ stands for the $n$-dimensional vector of controls (torques/forces). $G(q)$ is the $n$-dimensional vector of generalized gravity forces. $D(t, q, \dot{q})$ means the $n$-dimensional external disturbance signal which is (by assumption) at least absolutely continuous mapping with $\dot{D}(t, q, \dot{q})$ being a locally bounded Lebesgue measurable mapping. Moreover, $\|D\|$ and $\|\dot{D}\|$ are (by assumption) upper estimated as follows

$\|D\| \leq \alpha_{0}(t),\|\dot{D}\| \leq \alpha_{1}(t)$,

where $\alpha_{0}, \alpha_{1}$ stand for the known, non-negative functions. The general kinematic and differential mappings between joint coordinates $q$ and task coordinates $p=\left(p_{1}, \ldots, p_{m}\right)^{T} \in \mathbb{R}^{m}$ can be written as

$p=f(q, X), \quad \dot{p}=J(q, X) \dot{q}$,

where $n \geq m$ is the dimension of the Cartesian space in which the end-effector and manipulator operate; $f$ : $\mathbb{R}^{n} \times \mathbb{R}^{k} \longrightarrow \mathbb{R}^{m}$ denotes the $m$-dimensional nonlinear (with respect to $q$ ) mapping constructed from the kinematic equations of the manipulator; $J=\frac{\partial f}{\partial q}$ is the $m \times n$ Jacobian matrix; $X=\left(X_{1}, \ldots, X_{k}\right)^{T}$ stands for an ordered set of kinematic parameters such as link lengths and/or joint offsets; $k$ denotes the number of kinematic parameters. Moreover, there exist upper $X_{u}$ and lower $X_{l}$ bounds, respectively such that

$X_{l} \leq X \leq X_{u}$,

where inequality $\leq$ is defined component-wise. Due to the fact that kinematic redundancy is not significant in the design of our controller, the dimension of the task space is assumed without loss of generality in further analysis to be equal to $n$, i.e., $m=n$ (see comments of Remark 6 in the next section regarding the kinematically redundant mechanisms). A task accomplished by the robotic manipulator consists in tracking a desired trajectory $p_{d}(t) \in \mathbb{R}^{n}, t \in[0, \infty)$ which is assumed to be at least triply continuously differentiable, i.e., $p_{d}(\cdot) \in C^{3}[0, \infty)$. By introducing the task tracking error $e=\left(e_{1}, \ldots, e_{n}\right)^{T}=p-$ $p_{d}(t)=f(q, X)-p_{d}$, we may formally express the 
finite-time control problem by means of the following equations:

$\lim _{t \rightarrow T} e(t)=0, \lim _{t \rightarrow T} \dot{e}(t)=0, \lim _{t \rightarrow T} \ddot{e}(t)=0$,

where $0 \leq T$ denotes a finite-time of convergence of $f(q)$ to $p_{d}$, that is, $e(t)=\dot{e}(t)=\ddot{e}(t)=0$ for $t \geq T$. In the sequel, useful properties of Eq. 1 are summarised which will be utilised while designing the controller. The following inequalities are satisfied [8]:

$$
\begin{aligned}
& 0<\left\|M^{-1}\right\|_{F} \leq \Lambda_{\max },\|B\|_{F}+\|C\|_{F} \leq c_{1}, \\
& \|G\| \leq c_{2},
\end{aligned}
$$

where \|\|$_{F}$ means the Frobenius (Euclidean) matrix norm; $c_{1}, c_{2}, \Lambda_{\max }$ are known positive scalar coefficients. From Eqs. 6, 2 and 1, it follows that [8]

$\|\ddot{q}\| \leq \Lambda_{\max }\left(\|v\|+c_{1}\|\dot{q}\|^{2}+c_{2}+\alpha_{0}\right)$.

Moreover on account of Eq. 4 and definition of $J(q, X)$, the following inequality holds true for revolute kinematic pairs of a non-redundant manipulator:

$\|J\|_{F} \leq c_{3}$,

where $\|J\|_{F}=\max _{X_{l} \leq X \leq X_{u}}\left\{\|J(q, X)\|_{F}\right\} ; c_{3}$ is a known scalar coefficient. In order to obtain at least absolutely continuous control $v$, let us differentiate robot dynamic Eq. 1 with respect to time

$M(q) \frac{d^{3} q}{d t^{3}}+F(q, \dot{q}, \ddot{q}, t)=\dot{v}$,

where $F=\dot{M} \ddot{q}+\dot{B}(\dot{q} \cdot \dot{q})+\dot{C}\left(\dot{q}^{2}\right)+B \frac{d}{d t}(\dot{q} \cdot \dot{q})+$ $C \frac{d}{d t}\left(\dot{q}^{2}\right)+\dot{G}+\dot{D}$. Based on the derivative of dynamics (9), the next section will present an approach to the solution of the control problem (5) subject to Eqs. 1 and 9 making use of the Lyapunov stability theory.

\section{Control Design for the Robotic Manipulator}

Motivated in part by the static computed torque methodology $[8,19]$, we propose a new dynamically computed torque vector $\dot{v}$ of the form

$\dot{v}=\hat{J}^{T} \hat{M}(q) u+\hat{F}(q, \dot{q}, \ddot{q}, t)$,

where $\hat{J}=J(q, \hat{X})$ is the estimate of the Jacobian matrix $J=J(q, X) ; \hat{X}$ denotes the estimate of the unknown kinematic parameters $X ; X_{l} \leq \hat{X} \leq X_{u} ; u \in$ $\mathbb{R}^{n}$ is a new control to be determined; $\hat{\hat{M}}$ and $\hat{F}$ denote known estimates of the corresponding unknown terms
$M$ and $F$, respectively in dynamic Eq. 9. If $F$ is known mapping, we can take $\hat{F}=F$. Alternatively, $\hat{F}=$ 0 if no model of $F$ is available. Definition of $\hat{M}$ is given further on. In further analysis, $\hat{J}$ is assumed to be of the full rank in the operation region of the endeffector, i.e.,

$\operatorname{rank}(\hat{J})=n$.

Consequently, from Eq. 11, one also obtains that

$0<\lambda \mathbb{I}_{n} \leq \hat{J} M^{-1} \hat{J}^{T} \leq \Lambda \mathbb{I}_{n}$,

where $\lambda, \Lambda$ denote estimations of the minimal and maximal eigenvalues of matrix $\hat{J} M^{-1} \hat{J}^{T} ; \mathbb{I}_{n}$ stands for the $n \times n$ identity matrix. Let us note that condition (11) may be made somewhat more weaken. It suffices that for singular configuration $q^{\prime}$ the set $\left\{x^{\prime}: J^{T}\left(q^{\prime}, \hat{X}\right) x^{\prime} \neq 0 \wedge \operatorname{rank}\left(J^{T}\left(q^{\prime}, \hat{X}\right)\right)<n\right\}$ is non-empty. In other words, there exist $0 \neq x \in\left\{x^{\prime}\right.$ : $\left.J^{T}\left(q^{\prime}, \hat{X}\right) x^{\prime} \neq 0 \wedge \operatorname{rank}\left(J^{T}\left(q^{\prime}, \hat{X}\right)\right)<n\right\}$ such that $x \notin \operatorname{ker}\left(J^{T}\left(q^{\prime}, \hat{X}\right)\right)$. The use of Eq. 10 as a dynamic control law gives $M \frac{d^{3} q}{d t^{3}}+F=\hat{J}^{T} \hat{M} u+\hat{F}=\dot{v}$. Since $M$ is invertible, we obtain

$\frac{d^{3} q}{d t^{3}}=M^{-1} \hat{J}^{T} \hat{M} u+M^{-1}(\hat{F}-F)$

In the sequel, we introduce the following auxiliary matrix $\hat{\mathcal{R}} \in \mathbb{R}^{n \times n}$ equal to $\hat{\mathcal{R}}=\hat{J} M^{-1} \hat{J}^{T} \hat{M}$ which will play a crucial role in designing of our controller. Let us triply differentiate $e$ with respect to time to obtain

$\frac{d^{3} e}{d t^{3}}=J \frac{d^{3} q}{d t^{3}}+\ddot{J} \dot{q}+2 \dot{J} \ddot{q}-\frac{d^{3} p_{d}}{d t^{3}}$.

Inserting the right-hand side of Eq. 13 into Eq. 14, one obtains

$\frac{d^{3} e}{d t^{3}}=u+\left(\hat{\mathcal{R}}-\mathbb{I}_{n}\right) u+(J-\hat{J}) M^{-1} \hat{J}^{T} \hat{M} u+Q-\frac{d^{3} p_{d}}{d t^{3}}$,

where $Q=J M^{-1}(\hat{F}-F)+\ddot{J} \dot{q}+2 \dot{J} \ddot{q}$. Furthermore, based on definitions of $F$ in Eq. 9 and $Q$ in Eq. 15, an upper estimation on $\|Q\|$ takes the form

$\|Q\| \leq \mathcal{W}(t, q, \dot{q}, \ddot{q})$,

where $\mathcal{W}=c_{3} \Lambda_{\max }\|\hat{F}\|+c_{4}\|\dot{q}\|\|\ddot{q}\|+c_{5}\|\dot{q}\|^{3}+$ $c_{6}\|\dot{q}\|+c_{3} \Lambda_{\max } \alpha_{1} ; c_{4}, c_{5}$ and $c_{6}$ are (known by assumption) positive scalar coefficients for which the following inequalities hold true: $c_{4} \geq$ $\left\|J M^{-1}\right\|_{F}\left(\left\|\frac{\partial M}{\partial q}\right\|_{F}+\|B\|_{F}+\|C\|_{F}\right)+3\left\|\frac{\partial J}{\partial q}\right\|_{F}$; $c_{5} \geq\left\|J M^{-1}\right\|_{F}\left(\left\|\frac{\partial B}{\partial q}\right\|_{F}+\left\|\frac{\partial C}{\partial q}\right\|_{F}\right)+\left\|\frac{\partial^{2} J}{\partial q^{2}}\right\|_{F}$ and 
$c_{6} \geq\left\|J M^{-1}\right\|_{F}\left\|\frac{\partial G}{\partial q}\right\|_{F}$. Based on Eqs. 6 and 12, we can make the following remark:

$(\exists \hat{M}>0)(\exists \rho>0)\left(\left|\lambda_{\max }\left(\hat{\mathcal{R}}-\mathbb{I}_{n}\right)\right| \leq \rho<1\right)$,

where $\lambda_{\max }(\cdot)$ stands for the maximal eigenvalue of matrix $(\cdot)$. Let us note that it is not difficult to find matrix $\hat{M}$ fulfilling relations (17). If we set $\hat{M}=$ $\frac{2}{\Lambda+\lambda} \mathbb{I}_{n}$ then $\rho=\frac{\Lambda-\lambda}{\Lambda+\lambda}$ satisfies inequality (17). In the sequel, matrix $\hat{J}$ is assumed to fulfil the following inequality:

$0 \leq\|\hat{J}-J\|_{F} \leq \frac{\rho \cdot \eta}{\Lambda_{\max } \cdot\|J\|_{F} \cdot\|\hat{M}\|_{F}}$,

where $\eta$ is a gain coefficient; $\eta \in\left[0, \min \left\{1, \frac{1-\rho}{\rho}\right\}\right)$. Let us note that inequality (18) can be in practice easily fulfilled by selection of a sufficiently accurate device for measurement of kinematic parameters $X$ (link lengths, joint offsets). Before we propose our controller and show its properties, useful inequality will now be given.

Lemma 1 If $b_{i} \geq 0, i=1,2, \ldots, L$ and $L \geq 1$ then the following inequality holds true:

$\sum_{i=1}^{L} b_{i}^{\gamma} \geq\left(\sum_{i=1}^{L} b_{i}\right)^{\gamma}$

where $0<\gamma<1$ is a positive coefficient which takes for our purpose the form $\gamma=\frac{a}{b} ; a, b$ are positive odd numbers, $a<b<2 a$.

Proof The proof will be carried out based on the mathematical induction. For $L=1$ inequality (19) is obvious. In the next step $(L=2)$, we shall show that

$b_{1}^{\gamma}+b_{2}^{\gamma} \geq\left(b_{1}+b_{2}\right)^{\gamma}$.

Based on the properties of function $x^{\gamma}$ for $x \geq 0$, it is easily seen that

$1+x^{\gamma} \geq(1+x)^{\gamma}$.

Let us note that for $x=0$ and $0<\gamma<1$, inequality (21) holds true. The derivative of $1+x^{\gamma}$ for $x>0$ equals $\gamma \frac{1}{x^{1-\gamma}}$ and the derivative of $(1+x)^{\gamma}$ takes the form $\gamma \frac{1}{(1+x)^{1-\gamma}}$. Clearly, $\gamma \frac{1}{x^{1-\gamma}}>\gamma \frac{1}{(1+x)^{1-\gamma}}$. Consequently, we conclude that inequality (21) holds true for $x \geq 0$. Premultiplying both sides of inequality (21) by $b_{1}^{\gamma}$ (without loss of generality $b_{1}>0$ ) leads to the relation

$b_{1}^{\gamma}+\left(b_{1} x\right)^{\gamma} \geq\left(b_{1}+b_{1} x\right)^{\gamma}$.
Let us choose $x$ as $x=\frac{b_{2}}{b_{1}}$ and set it into Eq. 22. Consequently, inequality (20) is obtained. Let Lemma 1 be true for the induction step $L \geq 2$. Then for $L+1$ and from Eq. 19, we obtain

$$
\sum_{i=1}^{L+1} b_{i}^{\gamma}=\sum_{i=1}^{L} b_{i}^{\gamma}+b_{L+1}^{\gamma} \geq\left(\sum_{i=1}^{L} b_{i}\right)^{\gamma}+b_{L+1}^{\gamma} .
$$

Based on inequality (20), we finally have

$$
\sum_{i=1}^{L+1} b_{i}^{\gamma} \geq\left(\sum_{i=1}^{L+1} b_{i}\right)^{\gamma} \text {. }
$$

For arbitrary $\bar{a}=\left(a_{1}, \ldots, a_{L}\right)^{T}$ and $b_{i}=a_{i}^{2}$, $i=1, \ldots, L$, the following useful inequality is a consequence of Lemma 1:

$\left\|\bar{a}^{\gamma}\right\|^{2} \geq\|\bar{a}\|^{2 \gamma}$,

where $\bar{a}^{\gamma}=\left(a_{1}^{\gamma}, \ldots, a_{L}^{\gamma}\right)^{T}$. Let us observe, that Lemma 1 and its proof are somewhat different than Lemmas 1, 2 presented in [12]. In fact, inequality (19) was not proved in [12] for $L \geq 2$. Let $s=$ $\left(s_{1}, \ldots, s_{n}\right)^{T} \in \mathbb{R}^{n}$ be a task space sliding vector variable. In order to overcome the limitations and shortcomings of the first order classic sliding variables $[4,12,13]$, we propose the following non-singular terminal sliding manifold:

$s=\ddot{e}+\int_{0}^{t}\left(\lambda_{2} \ddot{e}^{3 / 5}+\lambda_{2} \lambda_{1}^{3 / 5}\left(\dot{e}^{9 / 7}+\lambda_{0}^{9 / 7} e\right)^{1 / 3}\right) d \tau$,

where $\lambda_{0}=\operatorname{diag}\left(\lambda_{0,1}, . ., \lambda_{0, n}\right) ; \lambda_{1}=$ $\operatorname{diag}\left(\lambda_{1,1}, . ., \lambda_{1, n}\right) ; \lambda_{2}=\operatorname{diag}\left(\lambda_{2,1}, . ., \lambda_{2, n}\right) ; \lambda_{i, j}$ stand for positive coefficients (controller gains); $i=0: 2 ; j=1: n$. The power of both $e, \dot{e}, \ddot{e}$ and $\lambda_{0}$, $\lambda_{1}, \lambda_{2}$ is defined component-wise. In what follows, we give a useful result [15].

Lemma 2 If $s=0$ then task errors $(e, \dot{e}, \ddot{e})$ converge in a finite-time to the origin $(e, \dot{e}, \ddot{e})=(0,0,0)$.

In order to fulfil equality constraints (5), a (simple) robust task space control law is proposed as follows

$u=u_{n}+u_{r}$, 
where

$u_{n}=\frac{d^{3} p_{d}}{d t^{3}}-\lambda_{2} \ddot{e}^{3 / 5}-\lambda_{2} \lambda_{1}^{3 / 5}\left(\dot{e}^{9 / 7}+\lambda_{0}^{9 / 7} e\right)^{1 / 3}-\Lambda_{s} s^{\beta}$,

$\Lambda_{s}=\operatorname{diag}\left(\Lambda_{s, 1} \ldots, \Lambda_{s, n}\right) ; \Lambda_{s, i}$ denote constant, positive controller gains; $\beta$ is defined similarly as $\gamma$, i.e., $\beta=\frac{a^{\prime}}{b^{\prime}} ; a^{\prime}, b^{\prime}$ are positive odd numbers, $a^{\prime}<b^{\prime}<$ $2 a^{\prime}$, and

$u_{r}= \begin{cases}-\frac{\kappa}{1-\rho} \frac{s}{\|s\|}\left(\rho\left\|u_{n}\right\|+\mathcal{W}\right) & \text { for } s \neq 0 \\ 0 & \text { otherwise, }\end{cases}$

$\kappa$ is a positive constant gain to be specified further on. Consequently, absolutely continuous control vector $v$ can be found by solving in the Filippov sense [6], the following differential equations with $u_{n}$ and $u_{r}$ given by Eqs. 26-27:

$\dot{v}=J^{T}(q, \hat{X}) \hat{M}\left(u_{n}+u_{r}\right)+\hat{F}(q, \dot{q}, \ddot{q}, t)$.

The existence of the solution of differential equation (28) is a consequence of the following simple remark. Based on discontinuous term (27), let us construct the Filippov mapping (a multi-valued function) $\Psi: \mathbb{R}^{n} \rightarrow 2^{\mathbb{R}^{n}}$ of the form $\Psi(s)=\frac{s}{\|s\|}$ for $s \neq 0$ and $\Psi(0)=\bar{B}(0,1)$ otherwise, where $\bar{B}(0,1)$ means the closed ball with centre $s=0$ and radius 1 . As is easy to see, $\Psi$ is upper semi-continuous what implies, based on [6], the existence of the solution of Eq. 28. Since the right-hand side of Eq. 28 is a non-Lipschitz and complex mapping (in fact, it is discontinuous), we assume in further analysis that the solution of the closed-loop system (1), (28) is unique. However, for the class of robot systems with both known inertia matrix $M(q)$ and prismatic joints, it is not difficult to conclude (using the maximal monotone property of multi-valued function $\Psi$ ) the uniqueness of the solution in forward time of Eqs. 1, 28. Diagonal matrices $\lambda_{0}, \lambda_{1}, \lambda_{2}$ of Eq. 28 affect the speed of the task error convergence to zero and gain matrix $\Lambda_{s}$ is responsible for the convergence of the sliding variable vector to zero. In numerical implementation, the elements of $\Lambda_{s}$ should be chosen sufficiently large in order to quickly attain sliding mode. In such a case, the closedloop is not especially sensitive to the choice of $\lambda_{0}$, $\lambda_{1}$ and $\lambda_{2}$. Let us observe that control law (25)-(28) requires on-line measurements of quantities $\dot{q}, \ddot{q}, \dot{e}$ and $\ddot{e}$, respectively which are assumed for a moment to be available. The aim is to provide conditions on controller gains $\lambda_{0}, \lambda_{1}, \lambda_{2}, \Lambda_{s}$ and $\kappa$, which guarantee fulfilment of equalities (5). Applying the Lyapunov stability theory, we now derive the following result.

Theorem 1 If $\hat{J}$ fulfils inequalities (17), (18) along desired trajectory $p_{d}$ and $\lambda_{0}, \lambda_{1}, \lambda_{2}, \Lambda_{s}>0, \kappa>$ $2 \frac{1-\rho}{1-\rho-\eta \rho}, 0 \leq \eta<\min \left\{1, \frac{1-\rho}{\rho}\right\}$ then control scheme (25)-(28) guarantees stable convergence in a finite time of the task space tracking errors $(e, \dot{e}, \ddot{e})$ to the $\operatorname{origin}(e, \dot{e}, \ddot{e})=(0,0,0)$.

Proof Consider the following Lyapunov function candidate:

$V=\frac{1}{2}\langle s, s\rangle$,

where $\langle$,$\rangle stands for the scalar product of vectors.$ Differentiating (29) with respect to time and taking into account definition (24) results in the following expression: $\dot{V}=\left\langle s, \frac{d^{3} e}{d t^{3}}+\lambda_{2} \ddot{e}^{3 / 5}+\lambda_{2} \lambda_{1}^{3 / 5}\left(\dot{e}^{9 / 7}+\right.\right.$ $\left.\left.\lambda_{0}^{9 / 7} e\right)^{1 / 3}\right\rangle$. Based on Eq. 15, one obtains that

$$
\begin{aligned}
\frac{d^{3} e}{d t^{3}}= & u_{n}+u_{r}+\left(\hat{\mathcal{R}}-\mathbb{I}_{n}\right)\left(u_{n}+u_{r}\right) \\
& +(J-\hat{J}) M^{-1} \hat{J}^{T} \hat{M}\left(u_{n}+u_{r}\right) \\
& +Q-\frac{d^{3} p_{d}}{d t^{3}} .
\end{aligned}
$$

Inserting the right-hand side of Eq. 30 into $\dot{V}$ results in

$$
\begin{aligned}
\dot{V}= & \left\langle s, u_{n}-\frac{d^{3} p_{d}}{d t^{3}}+\lambda_{2} \ddot{e}^{3 / 5}+\lambda_{2} \lambda_{1}^{3 / 5}\left(\dot{e}^{9 / 7}+\lambda_{0}^{9 / 7} e\right)^{1 / 3}\right\rangle \\
& +\left\langle s, u_{r}\right\rangle+\left\langle s,\left(\hat{\mathcal{R}}-\mathbb{I}_{n}\right)\left(u_{n}+u_{r}\right)\right. \\
& \left.+(J-\hat{J}) M^{-1} \hat{J}^{T} \hat{M}\left(u_{n}+u_{r}\right)+Q\right\rangle .
\end{aligned}
$$

Let us estimate the last term of $\dot{V}$. The estimation takes the form

$$
\begin{aligned}
& \left\langle s,\left(\hat{\mathcal{R}}-\mathbb{I}_{n}\right)\left(u_{n}+u_{r}\right)+(J-\hat{J}) M^{-1} \hat{J}^{T} \hat{M}\left(u_{n}+u_{r}\right)+Q\right\rangle \\
& \leq\|s\|\left(\rho\left\|u_{n}\right\|+\mathcal{W}\right)+\|s\| \rho\left\|u_{r}\right\|+\|s\| \| \mid J \\
& -\hat{J}\left\|_{F}\right\| M^{-1}\|\|_{F}\|\hat{J}\|\left\|_{F}\right\| \hat{M} \|_{F}\left(\left\|u_{n}\right\|+\left\|u_{r}\right\|\right) .
\end{aligned}
$$

Replacing $u_{r}$ in Eq. 32 by the right-hand side of Eq. 27 and taking into account the obvious inequality $\|\hat{J}\|_{F} \leq\|J\|_{F}$ (by definitions, we have $\hat{J}=$ $J(q, \hat{X})$ and $X_{l} \leq \hat{X} \leq X_{u}$. Consequently, 
$\|J\|_{F}=\max _{q} \max _{X_{l} \leq X \leq X_{u}}\left\{\|J(q, \quad X)\|_{F}\right\} \geq$ $\left.\max _{q}\left\{\|J(q, \hat{X})\|_{F}\right\}=\|\hat{J}\|_{F}\right)$, we have

$$
\begin{aligned}
& \left\langle s,\left(\hat{\mathcal{R}}-\mathbb{I}_{n}\right)\left(u_{n}+u_{r}\right)+(J-\hat{J}) M^{-1} \hat{J}^{T} \hat{M}\left(u_{n}+u_{r}\right)+Q\right\rangle \\
& \leq\|s\|\left(\rho\left\|u_{n}\right\|+\mathcal{W}\right)+\|s\| \frac{\rho \kappa}{1-\rho}\left(\rho\left\|u_{n}\right\|+\mathcal{W}\right)+ \\
& \|s\|\|J-\hat{J}\|_{F}\left\|M^{-1}\right\|_{F}\|J\|\left\|_{F}\right\| \hat{M} \|_{F}\left(\left\|u_{n}\right\|+\frac{\kappa}{1-\rho}\left(\rho\left\|u_{n}\right\|+\mathcal{W}\right)\right)
\end{aligned}
$$

Using assumption (18), one obtains after simple calculations the following inequality:

$$
\begin{aligned}
& \left\langle s,\left(\hat{\mathcal{R}}-\mathbb{I}_{n}\right)\left(u_{n}+u_{r}\right)+(J-\hat{J}) M^{-1} \hat{J}^{T} \hat{M}\left(u_{n}+u_{r}\right)+Q\right\rangle \\
& \leq\|s\|\left(\rho\left\|u_{n}\right\|+\mathcal{W}\right)\left(2+\frac{\rho \eta}{1-\rho}+\frac{\eta \rho \kappa}{1-\rho}\right) .
\end{aligned}
$$

Consequently, the sum of the last two terms in Eq. 31 may be upper estimated as follows

$$
\begin{aligned}
\left\langle s, u_{r}\right\rangle+ & \left\langle s,\left(\hat{\mathcal{R}}-\mathbb{I}_{n}\right)\left(u_{n}+u_{r}\right)\right. \\
+ & \left.(J-\hat{J}) M^{-1} \hat{J}^{T} \hat{M}\left(u_{n}+u_{r}\right)+Q\right\rangle \\
& \leq\|s\|\left(\rho\left\|u_{n}\right\|+\mathcal{W}\right) \\
& \times\left(-\frac{\kappa}{1-\rho}+2+\frac{\rho \kappa}{1-\rho}+\frac{\eta \rho \kappa}{1-\rho}\right) .
\end{aligned}
$$

Based on the assumptions of Theorem 1 for $\kappa$ and $\eta$, expression $-\frac{\kappa}{1-\rho}+2+\frac{\rho \kappa}{1-\rho}+\frac{\eta \rho \kappa}{1-\rho}$ in Eq. 35 is nonpositive. Hence, for arbitrary both $u_{n}$ and $\mathcal{W} \geq 0$ one obtains

$$
\begin{aligned}
\left\langle s, u_{r}\right\rangle & +\left\langle s,\left(\hat{\mathcal{R}}-\mathbb{I}_{n}\right)\left(u_{n}+u_{r}\right)\right. \\
& \left.+(J-\hat{J}) M^{-1} \hat{J}^{T} \hat{M}\left(u_{n}+u_{r}\right)+Q\right\rangle \leq 0 .
\end{aligned}
$$

Inserting the right-hand side of Eq. 26 into Eq. 31 and taking into account inequality (36) results in the following expression:

$$
\dot{V} \leq-\sum_{i=1}^{n} \Lambda_{s, i} s_{i}^{1+\beta} \leq-\min _{i}\left\{\Lambda_{s, i}\right\}\left\|s^{\frac{1+\beta}{2}}\right\|^{2} .
$$

Applying Eq. 23 to the last term of inequality (37) results in

$$
\begin{aligned}
\dot{V} & \leq-\min _{i}\left\{\Lambda_{s, i}\right\}\left(\sum_{i=1}^{n} s_{i}^{2}\right)^{\frac{1+\beta}{2}} \\
& =-\min _{i}\left\{\Lambda_{s, i}\right\} 2^{\frac{1+\beta}{2}} V^{\frac{1+\beta}{2}} .
\end{aligned}
$$

Since $\min _{i}\left\{\Lambda_{s, i}\right\}>0$, expression (38) proves that TSM $s=0$ is attainable in a finite time less than or equal to $\frac{2 V(0)^{\frac{1-\beta}{2}}}{\min _{i}\left\{\Lambda_{s, i}\right\}(1-\beta) 2^{\frac{1+\beta}{2}}}$. Consequently, from

Lemma 1, it follows that the origin $(e, \dot{e}, \ddot{e})=$ $(0,0,0)$ is attainable in a finite time $T$.
A few remarks may be made regarding the control law (25)-(28) and Theorem 1.

- Remark 1. Observe that controller gain of $u_{r}$ given by Eq. 27 is a feed-back adjustable function equal to $\rho\left\|u_{n}\right\|+\mathcal{W}$. The control laws known from the literature (see e.g. $[1-3,5,8,19]$ ) require boundedness of $\dot{q}$ which implies large controller gains to cope with the uncertainty over the whole operation region.

- Remark 2. It is also worth to notice that our feedback adjustable amplitude term $\frac{\kappa}{1-\rho}\left(\rho\left\|u_{n}\right\|+\right.$ $\mathcal{W})$ makes it possible to cope with globally unbounded uncertainties. In general, in that case, only local uncertainty suppression is available in the literature for multi-input systems. In such a context, a class of gain-function robust controllers with single input and adjustable amplitude was recently proposed in works $[9,11]$ to overcome globally unbounded uncertainty problem.

- Remark 3. Let us note that expression (28) presents a transposed Jacobian controller. In such a context, the use of the transpose of the Jacobian is a well-known technique and there are several papers [52-57] that include its stability analysis. Nevertheless, work [52] guarantees only ultimate boundedness provided that $\dot{p}_{d}(t)$ is globally norm bounded. The asymptotically stable purely kinematic control scheme offered in [53] indeed eliminates explicit computation of the inverse but introduces undesirable chattering effect. In work [55], it is claimed that controllers based on transposed Jacobian and inverse Jacobian are dual in the sense that the transformation from task space to joint space can be either defined as transposed Jacobian or inverse Jacobian. As was also shown in [55, 56], approximate transpose Jacobian control law is asymptotically stable. In work [57], a modified transpose Jacobian algorithm was developed which employs stored data of the control command in the previous time step, as a learning tool to yield an improved performance. However, works [54-57] have shown stability of the performance for the set-point control problems. On the other hand, Theorem 1 provides stability analysis for the trajectory tracking problems. Moreover, chattering-free transposed Jacobian controller (28) is able to attain the stable equilibrium $(e, \dot{e}, \ddot{e})=(0,0,0)$ in a finite time. Due to involving the sliding mode term $u_{r}$, 
controller (28) is also robust against uncertainties of both kinematic and dynamic equations and external unbounded disturbances.

- Remark 4. Let us note that term $\left\langle s, \hat{\mathcal{R}} u_{r}\right\rangle$ in Eq. 31 can be transformed after simple calculations as follows $\left\langle s, \quad \hat{\mathcal{R}} u_{r}\right\rangle=$ $-\kappa\left\langle\hat{J}^{T} s, M^{-1} \hat{J}^{T} s\right\rangle \frac{2}{\|s\|(\Lambda+\lambda)}\left(\rho\left\|u_{n}\right\|+\mathcal{W}\right)$. Let $\hat{J}$ be singular at manipulator configuration $q^{\prime}$ and $0 \neq s \notin \operatorname{ker}\left(J^{T}\left(q^{\prime}, \hat{X}\right)\right)$. Hence, for sufficiently large $\kappa$, term $\left\langle s, \hat{\mathcal{R}} u_{r}\right\rangle$ can take arbitrarily large negative values which implies negative value of time derivative $\dot{V}$. Consequently, controller (25)-(28) is able to generate manipulator motions which can pass through singular manifold $\left\{q^{\prime}\right.$ : $\left.\operatorname{det}\left(J\left(q^{\prime}, \hat{X}\right)\right)=0\right\}$.

- Remark 5. Observe that the performance improvement of controller (28) is achieved by an increase of control contribution in Eq. 26 for small tracking errors, because for $0<\beta<1$ and $\left|s_{i}(t)\right|<1$ we have $\left|s_{i}(t)\right|^{\beta}>\left|s_{i}(t)\right|$. Also, when the tracking error is large (especially at the beginning of the control process), the terminal sliding mode controller (28) gives smaller control effort than that resulting from a linear sliding mode since $\left|s_{i}(t)\right|^{\beta}<\left|s_{i}(t)\right|$ for $\left|s_{i}(t)\right|>1$. The same is true for the term $e$ in Eq. 24. This remark is also confirmed by numerical simulations carried out in the next section.

- Remark 6. Observe that vector $p$ from Eq. 3 contains, in general, conventional end-effector coordinates (its location and orientation) and possibly additional user specified coordinates (introduced in $[43,51])$ to fulfil useful goals: a singularity avoidance, posture control, obstacle avoidance, etc. [49, 50] provided that $m<n$. Consequently, a kinematically redundant mechanism becomes non-redundant after augmenting the $m$ dimensional Cartesian space, in which the endeffector operates, by $n-m$ additional user specified coordinates.

- Remark 7. Let us note that for the particular case $\eta=0$, i.e., when Jacobian matrix fulfils equality $\hat{J}=J$ (kinematic parameters are fully known, i.e., $\hat{X}=\mathrm{X}$ ), condition on $\kappa$ in Theorem 1 takes the form $\kappa>2$. This inequality is consistent with a stronger one derived in our recent work [70], in which a simpler technique of the proof was applied resulting in $\kappa>1$.
In most cases, real robotic manipulators are equipped with encoders which measure only joint positions and/or task errors. Hence, reconstruction or estimation of joint velocity, joint acceleration, task error velocity and task error acceleration is required to apply controller (28). High-frequency measurement noise related with application of backward difference estimation or additional phase lag introduced by the filtering techniques [58] may be harmful to the closed-loop system (1), (28). Recently, a method of direct lag-free joint acceleration sensing has been proposed in work [59]. However, its combination with our controller requires further investigations. Application of Luenberger-style observers [60, 61], high-gain observers [62,63], model-free observers [64, 65] or a class of observers based on the sliding-mode algorithms [66] seems to be an efficient approach compared with both backward difference technique and direct measurement. Although all the aforementioned observers are able to reliably reconstruct manipulator state (both joint velocity and acceleration) based on position measurement, there appears a difficulty to combine our control law and the observer from [60-65]. In order to make such combination possible, observers proposed in works [60-65] have to satisfy the so-called separation principle [68] which implies both the continuity of the controllers from [60-65] with the fully available state and asymptotic stability of the closed-loop system under the continuous state feed-back controllers. Let us observe that our control law (28) is discontinuous what prevents an application of the state observers from [60-65]. Although the observer offered in [66] fulfils the separation principle, our controller handles unbounded uncertainties (in dynamics and disturbances) and does not require boundedness of $\dot{q}$ and $\ddot{q}$, respectively. A computationally efficient approach based on the uniform robust exact finite-time differentiation has been recently proposed in works $[10,11]$ to numerically find derivatives of absolutely continuous functions. The separation principle is trivially fulfilled for differentiators (model-free observers) from [10, 11]. Assuming that position $q=q(t)$ and task error $e=e(t)$ are known (measurable), one can exactly reconstruct joint velocity $\dot{q}(t)$, joint acceleration $\ddot{q}(t)$, task error velocity $\dot{e}(t)$ and task error acceleration $\ddot{e}(t)$ (by neglecting the measurement noise of a device) after finite-times of transient processes, say $T_{q}^{\prime}, T_{e}^{\prime}>0$, respectively. The second-order uniform robust exact differentiators 
(model-free observers) take in our case the following forms:

$$
\begin{aligned}
& \dot{y}_{0}=y_{1}-\hat{\lambda}_{2}^{q} L_{q}(t)^{1 / 3}\left|y_{0}-q\right|^{2 / 3} \operatorname{sign}\left(y_{0}-q\right), \\
& \dot{y}_{1}=y_{2}-\hat{\lambda}_{1}^{q} L_{q}(t)^{2 / 3}\left|y_{0}-q\right|^{1 / 3} \operatorname{sign}\left(y_{0}-q\right), \\
& \dot{y}_{2}=-\hat{\lambda}_{0}^{q} L_{q}(t) \operatorname{sign}\left(y_{0}-q\right),
\end{aligned}
$$

and

$$
\begin{aligned}
& \dot{z}_{0}=z_{1}-\hat{\lambda}_{2}^{e} L_{e}(t)^{1 / 3}\left|z_{0}-e\right|^{2 / 3} \operatorname{sign}\left(z_{0}-e\right), \\
& \dot{z}_{1}=z_{2}-\hat{\lambda}_{1}^{e} L_{e}(t)^{2 / 3}\left|z_{0}-e\right|^{1 / 3} \operatorname{sign}\left(z_{0}-e\right), \\
& \dot{z}_{2}=-\hat{\lambda}_{0}^{e} L_{e}(t) \operatorname{sign}\left(z_{0}-e\right),
\end{aligned}
$$

where $\hat{\lambda}_{0}^{q}, \hat{\lambda}_{1}^{q}, \hat{\lambda}_{2}^{q}, \hat{\lambda}_{0}^{e}, \hat{\lambda}_{1}^{e}, \hat{\lambda}_{2}^{e}$ are positive constants whose values were suggested in $[10,11] ; y_{0}(t), y_{1}(t)$, $y_{2}(t), z_{0}(t), z_{1}(t), z_{2}(t) \in \mathbb{R}^{n} \cdot y_{1}, y_{2}, z_{1}, z_{2}$ denote the outputs of differentiators (39)-(40) reconstructing exactly joint velocity $\dot{q}(t)$, joint acceleration $\ddot{q}(t)$, task error velocity $\dot{e}(t)$, task error acceleration $\ddot{e}(t)$, i.e., $\dot{q}(t)=y_{1}(t), \ddot{q}(t)=y_{2}(t)$ for $t \geq T_{q}^{\prime}, \dot{e}(t)=$ $z_{1}(t), \ddot{e}(t)=z_{2}(t)$ for $t \geq T_{e}^{\prime} . L_{q}(t), L_{e}(t)$ stand for positive continuous functions which take (based on Eq. 7, (25)-(28)) the forms $L_{q}(t)=L^{\prime}(t)+$ $L^{\prime \prime}(t)$, where $L^{\prime}=\Lambda_{\max }\left[c_{3} \Lambda\left(\left\|u_{n}\right\|+\frac{\kappa}{1-\rho}\left(\rho\left\|u_{n}\right\|+\right.\right.\right.$ $\mathcal{W}))+\|\hat{F}\|] ; L^{\prime \prime}=\Lambda_{\max }\left\{\left\|y_{1}\right\|\left(\left\|\frac{\partial M}{\partial q}\right\|_{F}+\|B\|_{F}+\right.\right.$ $\left.\|C\|_{F}\right) \Lambda_{\max }\left[\|v\|+c_{1}\left\|y_{1}\right\|^{2}+c_{2}+\alpha_{0}(t)\right]+\left(\left\|\frac{\partial B}{\partial q}\right\|_{F}+\right.$ $\left.\left.\left\|\frac{\partial C}{\partial q}\right\|_{F}\right)\left\|y_{1}\right\|^{3}+\left\|\frac{\partial G}{\partial q}\right\|_{F}\left\|y_{1}\right\|+\alpha_{1}(t)\right\}$ and $L_{e}(t)=$ $c_{3} L_{q}(t)+c_{4}\left\|y_{1}\right\|\left\|y_{2}\right\|+c_{5}\left\|y_{1}\right\|^{3}+\left\|\ddot{p}_{d}\right\|$. The quantities $L_{q}(t), L_{e}(t)$ represent physically upper estimations of the norms of $\frac{d^{3} q}{d t^{3}}, \frac{d^{3} e}{d t^{3}}$ (manipulator joint and task error jerks). Let us define concatenating control $v_{c}=\left(v_{c, 1}, \ldots, v_{c, n}\right)^{T}$ as follows

$v_{c}= \begin{cases}v^{\prime}(t), & t \in\left[0, \max \left\{T_{q}^{\prime}, T_{e}^{\prime}\right\}\right], \\ v(t) & \text { given by Eq. } 28, \dot{q}=y_{1}, \ddot{q}=y_{2}, \dot{e}=z_{1}, \ddot{e}=z_{2}, t \geq \max \left\{T_{q}^{\prime}, T_{e}^{\prime}\right\},\end{cases}$

where $v^{\prime}(t)$ is arbitrary absolutely continues mapping of time $t$ (e.g. $v^{\prime}(t)=0$ ). Based on Eqs. 39-40 and 41 , we are now in position to give the following theorem.

Theorem 2 If $q$, e are only available from measurements, $\hat{J}$ fulfils inequalities (17), (18) along desired trajectory $p_{d}$ and $\lambda_{0}, \lambda_{1}, \lambda_{2}, \Lambda_{s}>0, \kappa>2 \frac{1-\rho}{1-\rho-\eta \rho}$, $0 \leq \eta<\min \left\{1, \frac{1-\rho}{\rho}\right\}$ then control scheme (41) guarantees stable convergence in a finite time of the task space tracking errors $(e, \dot{e}, \ddot{e})$ to the origin $(e, \dot{e}, \ddot{e})=(0,0,0)$.

Proof Inserting $v^{\prime}$ into dynamic Eq. 1 results in measured joint positions $q=q(t)$ and task errors $e=$ $e(t)$ which serve as inputs to differentiators (39)-(40). For $t>\max \left\{T_{q}^{\prime}, T_{e}^{\prime}\right\}$, one obtains $\dot{q}(t)=y_{1}(t)$, $\ddot{q}(t)=y_{2}(t), \dot{e}(t)=z_{1}(t)$ and $\ddot{e}(t)=z_{2}(t)$, respectively. Hence, control $v(t)$ defined by Eqs. 25-28 can be applied with initial conditions $v\left(T^{\prime}\right)=v^{\prime}\left(T^{\prime}\right)$ and $\dot{q}\left(T^{\prime}\right)=y_{1}\left(T^{\prime}\right), \ddot{q}\left(T^{\prime}\right)=y_{2}\left(T^{\prime}\right), \dot{e}\left(T^{\prime}\right)=z_{1}\left(T^{\prime}\right)$, $\ddot{e}\left(T^{\prime}\right)=z_{2}\left(T^{\prime}\right)$ to track $p_{d}$, where $T^{\prime} \geq \max \left\{T_{q}^{\prime}, T_{e}^{\prime}\right\}$. From Theorem 1, it follows that $s=0$ is attainable in a finite time less than or equal to $\frac{2 V\left(T^{\prime}\right)^{\frac{1-\beta}{2}}}{\min _{i}\left\{\Lambda_{s, i}\right\}(1-\beta) 2^{\frac{1+\beta}{2}}}$. Finally, from Lemma 1, it follows that the origin $(e, \dot{e}, \ddot{e})=(0,0,0)$ is attained in a finite time.

\section{Numerical Simulations}

This section demonstrates the performance of the controllers given by expressions (28), (41) on a selected

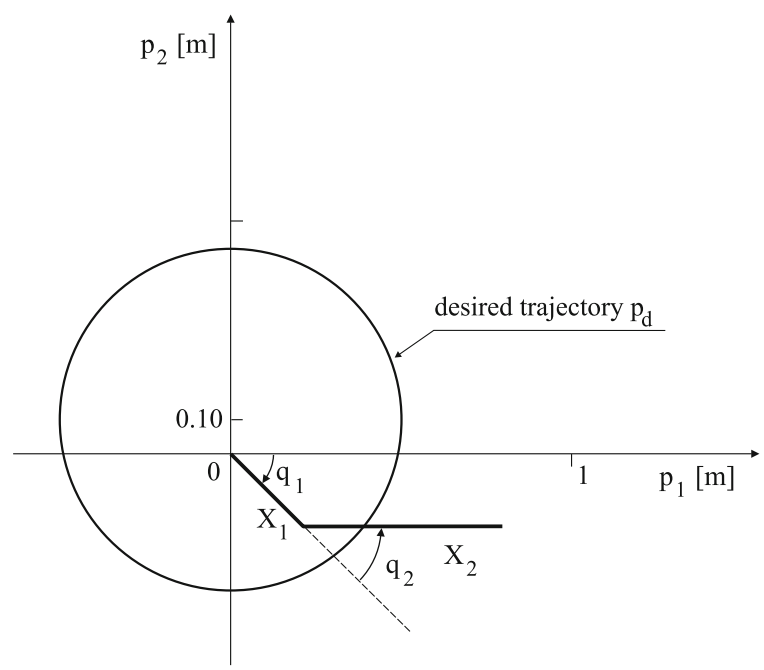

Fig. 1 A kinematic scheme of the manipulator and the task to be accomplished 
Fig. 2 Task error $e_{1}$ for controller (41) with linearly parametrizable continuous friction terms

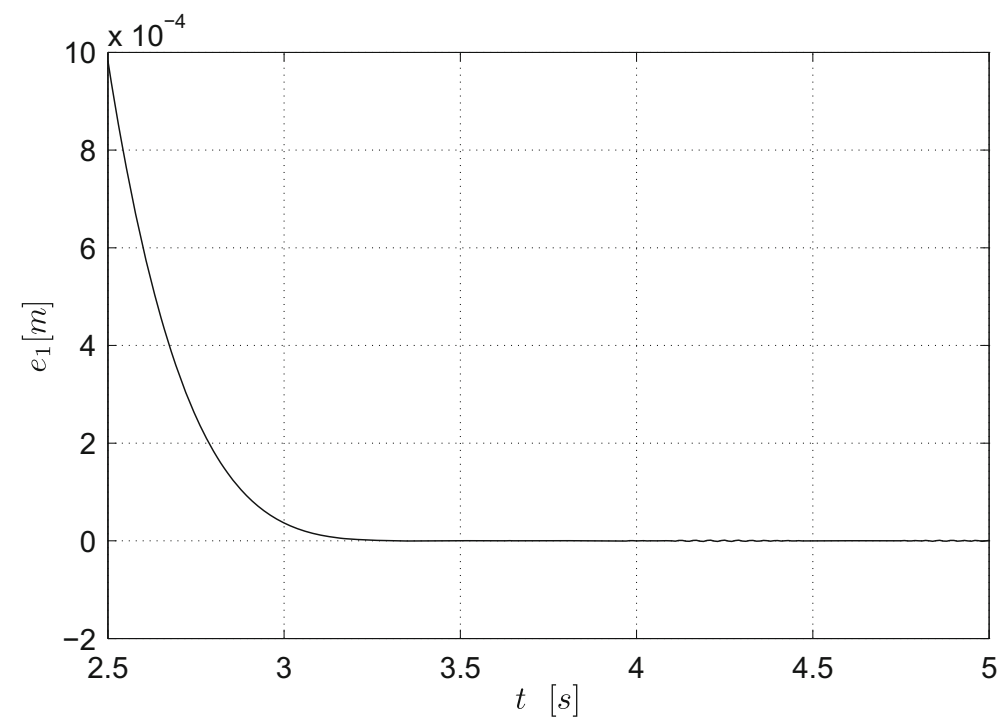

manipulator task. For this purpose, we utilise the data of the Experimental Direct Drive Arm (EDDA manipulator) $(n=2)$ operating in the two-dimensional task space $(m=2)$ whose kinematic scheme is shown in Fig. 1, where $X=\left(X_{1}, X_{2}\right)^{T}$ stands for the ordered set of link lengths. The model of EDDA manipulator used in this Section has been borrowed from the book [73] (see expressions (5.21)-(5.24)). In the simulations, SI units are used. The (nominal) link lengths are assumed to be equal to $X_{n m}=\left(\begin{array}{ll}0.30 .58\end{array}\right)^{T}$. The estimation term $\hat{X}$ takes in all the simulations the form as follows $\hat{X}=\left(\begin{array}{lll}0.32 & 0.53\end{array}\right)^{T}$. The components of dynamic equations of this manipulator are as follows $M=\left[\begin{array}{cc}\theta_{1}+0.6 \theta_{4} \cos \left(q_{2}\right) & \theta_{3}+0.3 \theta_{4} \cos \left(q_{2}\right) \\ \theta_{3}+0.3 \theta_{4} \cos \left(q_{2}\right) & \theta_{3}\end{array}\right] ; B=$ $\left[\begin{array}{c}-1 \\ 0\end{array}\right] 0.3 \theta_{4} \sin \left(q_{2}\right) ; C=\left[\begin{array}{cc}1 & -1 \\ 1 & 0\end{array}\right] 0.3 \theta_{4} \sin \left(q_{2}\right) ;$ $G=g\left(\begin{array}{c}\theta_{2} \cos \left(q_{1}\right)+\theta_{4} \cos \left(q_{1}+q_{2}\right) \\ \theta_{4} \cos \left(q_{1}+q_{2}\right)\end{array}\right) ; g$ stands for the gravitational acceleration; parameters $\theta_{i}, i=1$ : 4 take the following nominal values: $\theta_{1, n m}=3.1$, $\theta_{2, n m}=9.5, \theta_{3, n m}=0.24, \theta_{4, n m}=0.77$, i.e., $\Theta_{n m}=\left(\begin{array}{llll}3.1 & 9.5 & 0.24 & 0.77\end{array}\right)^{T}$. In order to numerically compare our controller with those known from the
Fig. 3 Task error $e_{2}$ for controller (41) with linearly parametrizable continuous friction terms

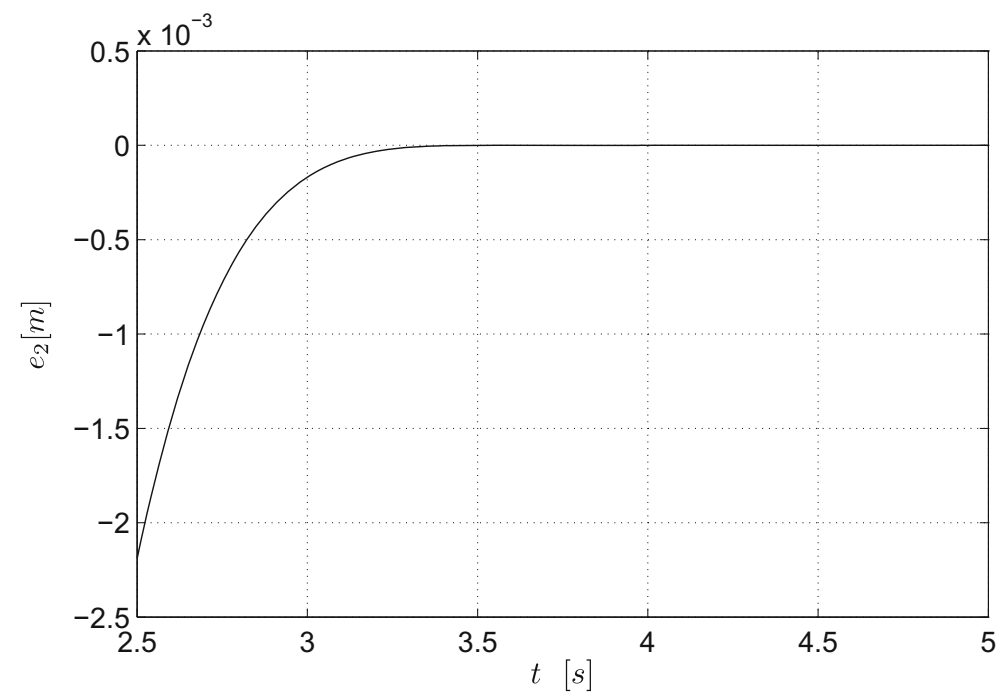


Fig. 4 Torque $v_{c, 1}$ for controller (41) with linearly parametrizable continuous friction terms

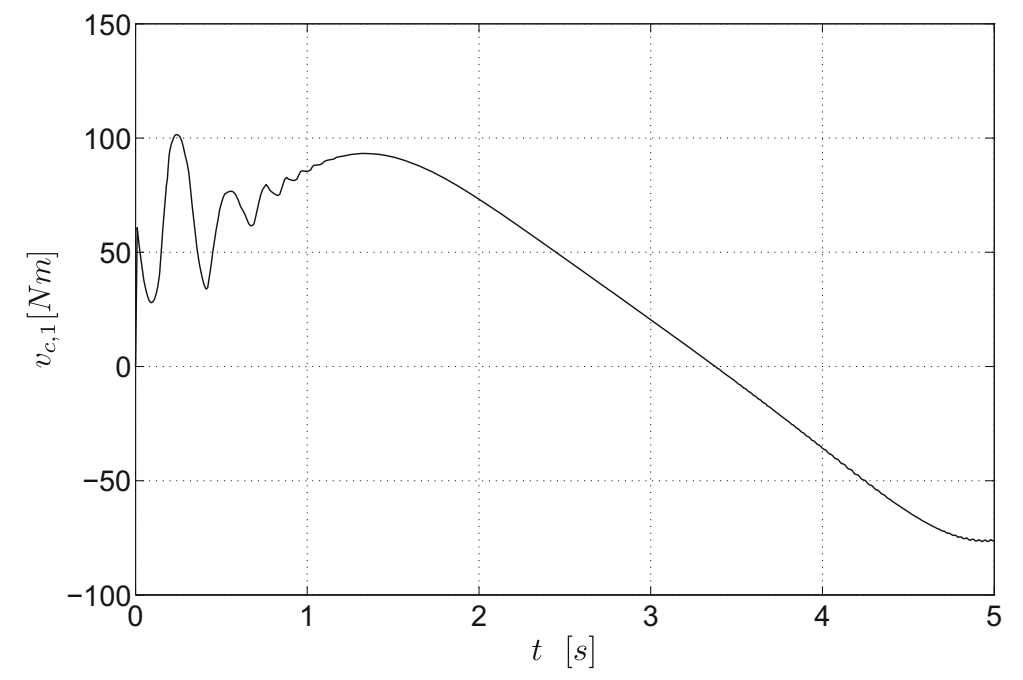

literature, we reformulate dynamic Eq. 1 to a partially linearly parametrizable form as follows [8].

$M \dot{r}+E(q, \dot{q}) r+Y(e, \dot{e}, q, \dot{q}) \Theta+D(t, q, \dot{q})=v$, where $E=\left[\begin{array}{cc}\dot{q}_{1} & -\left(\dot{q}_{1}+\dot{q}_{2}\right) \\ \dot{q}_{1} & 0\end{array}\right] 0.3 \theta_{4} \sin \left(q_{2}\right)$; $r=\dot{q}-J^{-1}\left(\dot{p}_{d}-\alpha e\right)$ denotes a filtered tracking error signal [38, 39]; $Y=Y(e, \dot{e}, q, \dot{q}) \in \mathbb{R}^{2 \times 4}$ is the regression matrix; $Y \Theta=M \frac{d}{d t} J^{-1}\left(\dot{p}_{d}-\alpha e\right)+$ $E J^{-1}\left(\dot{p}_{d}-\alpha e\right)+G+D ; \Theta=\left(\theta_{1}, \ldots, \theta_{4}\right)^{T} ; \alpha$ stands for a constant gain coefficient. The initial configuration and velocity of the manipulator are equal in all the experiments to $q(0)=(-\pi / 4, \pi / 4)^{T}$,
$\dot{q}(0)=(0,0)^{T}$, respectively. Due to the planar manipulator of the two revolute kinematic pairs taken for simulations, there exists analytic solution to the inverse kinematics problem, i.e., $q=f^{-1}\left(p_{d}(t)\right), t \in[0, \infty), X=X_{n m}$, where $f^{-1}$ is given analytically. Hence, based on the nominal dynamic parameters $\Theta_{n m}$, we may numerically find the estimate of $\Lambda_{\max }$ from the following optimisation task: $\Lambda_{\max }=$ $\left.\max _{t \in[0, \infty)}\left\{|| M^{-1}(q) \|_{F}\right\}\right|_{\left(\Theta=\Theta_{n m}, q=f^{-1}\left(p_{d}(t)\right)\right)}$. To simplify the computations, our estimates for controller (41) are chosen as $\hat{M}=\frac{2}{\lambda+\Lambda} \mathbb{I}_{2} ; \hat{F}=0$; $\lambda=0.17 ; \Lambda=\Lambda_{\max }=5$. In order to speed up the convergence process of differentiators (39)-(40),
Fig. 5 Torque $v_{c, 2}$ for controller (41) with linearly parametrizable continuous friction terms

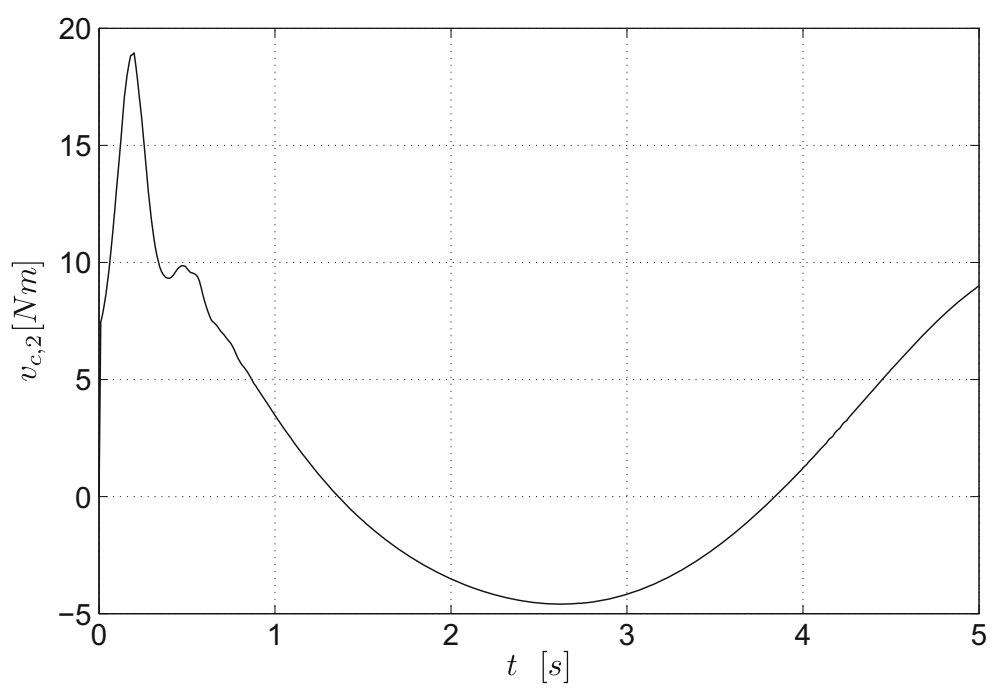


Fig. 6 Task error $e_{1}$ for controller (42) with linearly parametrizable continuous friction terms

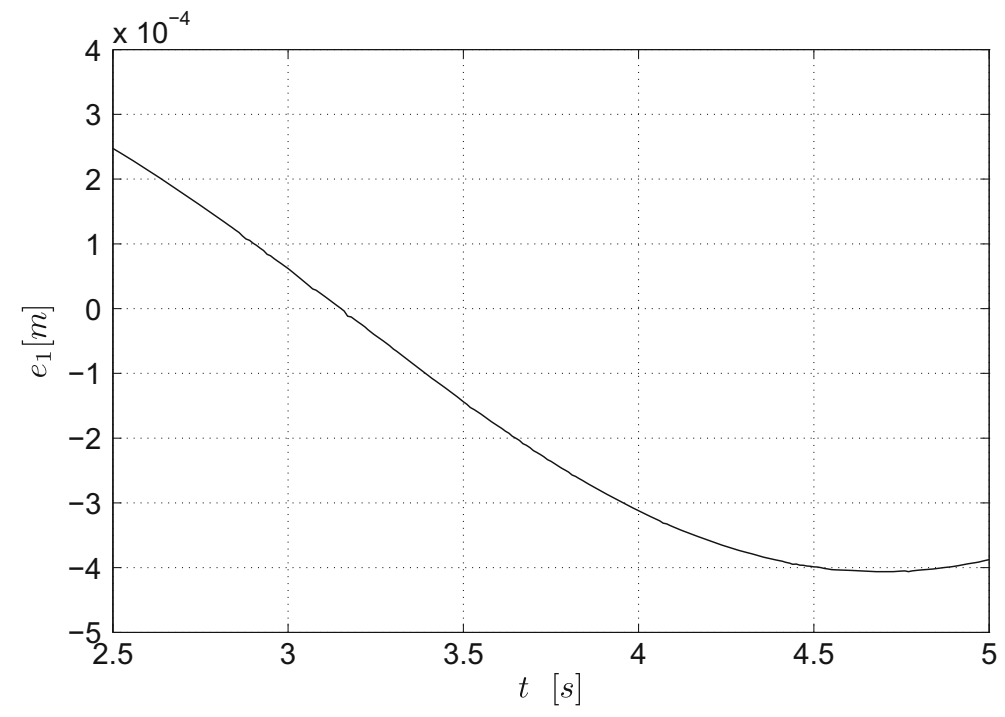

we have chosen good initial guesses $y_{1}(0), y_{2}(0)$, $z_{1}(0), z_{2}(0)$ in the numerical examples (which imply relation $T^{\prime} \simeq \max \left\{T_{q}^{\prime}, T_{e}^{\prime}\right\} \simeq 0$ - see the proof of Theorem 2) based on the nominal values of our kinematic and dynamic models. Consequently, differentiators (39)-(40) were run with the following initial values: $y_{1}(0)=\dot{q}(0), y_{2}(0)=(-22.1,5.7)^{T}$, $z_{1}(0)=(0,-0.5)^{T}, z_{2}(0)=(-4.19,-14.2)^{T}$, $v(0)=(0,0)^{T}$ and parameters $\hat{\lambda}_{0}^{q}=\hat{\lambda}_{0}^{e}=13.2$; $\hat{\lambda}_{1}^{q}=\hat{\lambda}_{1}^{e}=16.8 ; \hat{\lambda}_{2}^{q}=\hat{\lambda}_{2}^{e}=24$, respectively. Due to conservative nature of estimates $L_{q}$ and
$L_{e}$ in (39)-(40), they are assumed for simplicity of computations in all the simulations to be equal, i.e., $L_{e}(t)=L_{q}(t)$. The estimates of the constants $c_{4}, c_{5}$ and $c_{6}$ can be determined as follows $c_{4} \geq \max _{t \in[0, \infty)}\left\{\left\|J M^{-1}\right\|_{F}\left(\left\|\frac{\partial M}{\partial q}\right\|_{F}+\right.\right.$ $\left.\left.\|B\|_{F}+\|C\|_{F}\right)+\left.3\left\|\frac{\partial J}{\partial q}\right\|\right|_{F}\right\}\left.\right|_{\left(\Theta=\Theta_{n m}, q=f^{-1}\left(p_{d}(t)\right)\right)} ;$ $c_{5} \geq \max _{t \in[0, \infty)}\left\{\left\|J M^{-1}\right\|_{F}\left(\left\|\frac{\partial B}{\partial q}\right\|_{F}+\left\|\frac{\partial C}{\partial q}\right\|_{F}\right)+\right.$ $\left.\left\|\frac{\partial^{2} J}{\partial q^{2}} \mid\right\|_{F}\right\}\left.\right|_{\left(\Theta=\Theta_{n m}, q=f^{-1}\left(p_{d}(t)\right)\right)}$ and estimate of $c_{6}$ as $c_{6}$ $\geq\left.\max _{t \in[0, \infty)}\left\{\left\|J M^{-1}\right\|_{F}\left\|\frac{\partial G}{\partial q}\right\|_{F}\right\}\right|_{\left(\Theta=\Theta_{n m}, q=f^{-1}\left(p_{d}(t)\right)\right)}$. Nevertheless, in order to simplify numerical compu-
Fig. 7 Task error $e_{2}$ for controller (42) with linearly parametrizable continuous friction terms

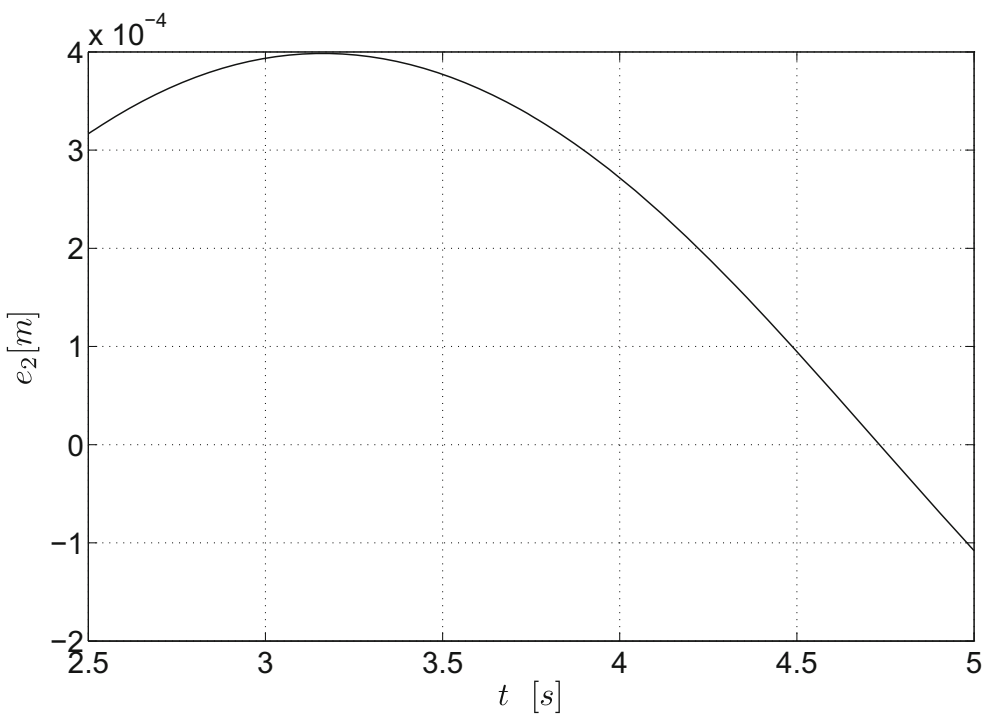


Fig. 8 Torque $v_{1}$ for controller (42) with linearly parametrizable continuous friction terms

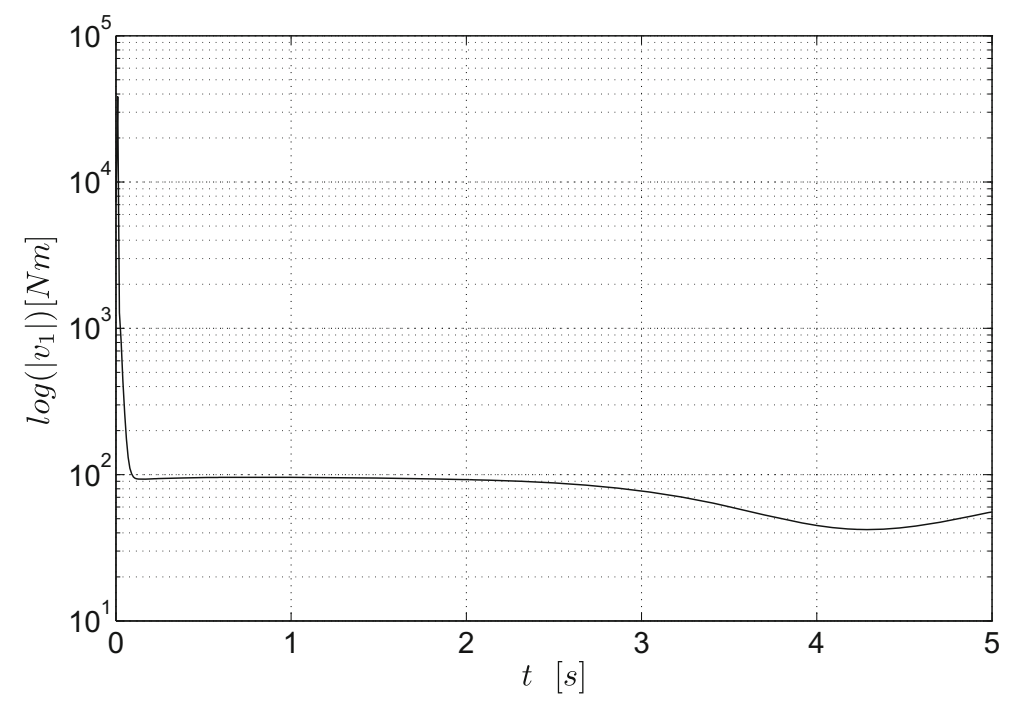

tations, rough conservative estimates of $c_{i}, i=1: 6$ have been assumed. Hence, positive constant coefficients $c_{i}, i=1: 6$ were chosen as follows $c_{1}=10, c_{2}=150, c_{3}=1, c_{4}=27, c_{5}=5$ and $c_{6}=10$, respectively. The aim of the controller (41) is to track by the end-effector a circle trajectory, expressed by the following equation: $p_{d}(t)=(0.5 \cos (t), 0.1+0.5 \sin (t))^{T}$. Disturbing term $D$ is assumed in this simulation to represent both the friction in the sliding (viscous) and in the presliding (also called stiction) regime [48, 69] of the form $D=\theta_{5}(q-q(0))+\theta_{6} \dot{q}$ with the nominal values of parameters $\theta_{5}, \theta_{6}$ equal to $\theta_{5, \mathrm{~nm}}=\theta_{6, \mathrm{~nm}}=2$. Moreover, $\dot{D}$ is equal to $\dot{D}=\theta_{5} \dot{q}+\theta_{6} \ddot{q}$. The control law proposed in $[38,39]$ for linearly parametrizable continuous term $D$ is given by the following expression:

$v=Y \hat{\Theta}+K r-J^{T} e+\hat{\theta}_{5}(q-q(0))+\hat{\theta}_{6} \dot{q}$, where $K$ denotes a gain coefficient; $\hat{\Theta}=$ $\left(\hat{\theta}_{1}, \ldots, \hat{\theta}_{4}\right)^{T}, \hat{\theta}_{5}, \hat{\theta}_{6}$ mean the best guess estimates of the unknown parameters $\Theta, \theta_{5}$ and $\theta_{6}$. In order to exhibit the role of feed-back amplitude adjustable term $\frac{\kappa}{1-\rho}\left(\rho\left\|u_{n}\right\|+\mathcal{W}\right)$ from (27) compared with constant gains $\hat{\Theta}, \hat{\theta}_{5}, \hat{\theta}_{6}$ of (42), estimates $\hat{\Theta}, \hat{\theta}_{5}$ and $\hat{\theta}_{6}$
Fig. 9 Torque $v_{2}$ for controller (42) with linearly parametrizable continuous friction terms

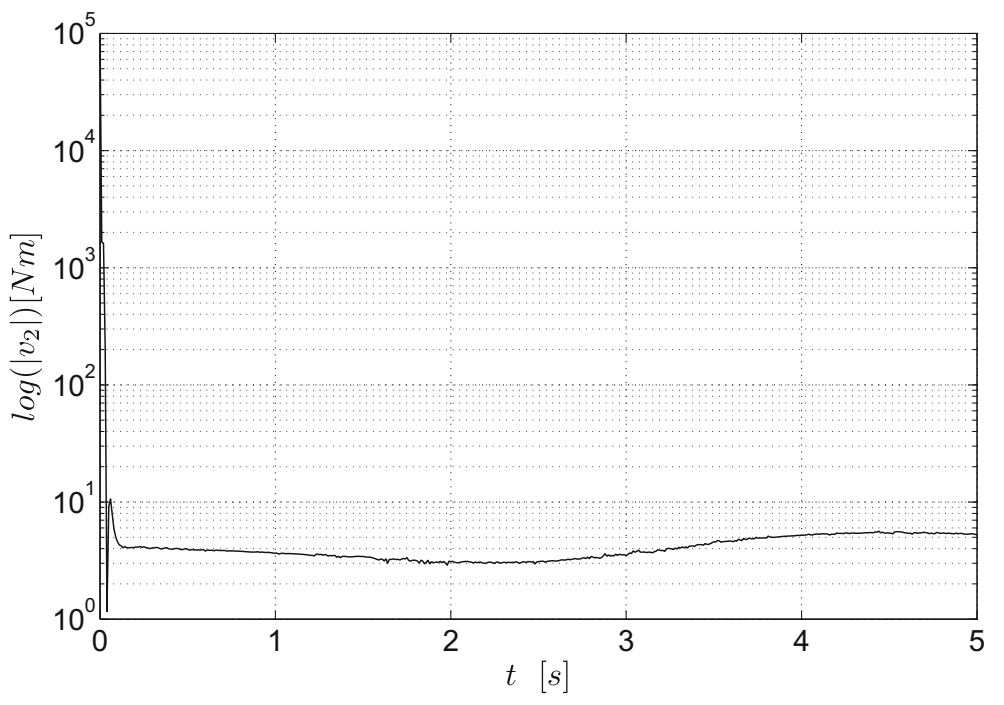


Fig. 10 Task error $e_{1}$ for controller (41) with both linearly and non-linearly parametrizable discontinuous friction terms

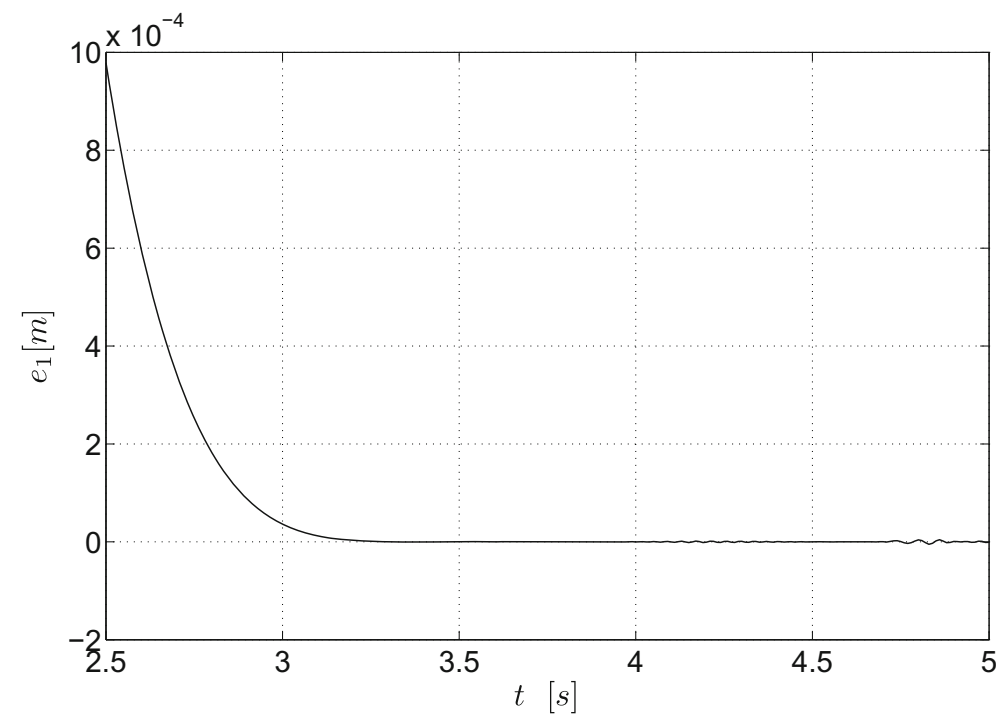

are assumed in this experiment to be fully known, i.e., the following equalities are now fulfilled: $\hat{\Theta}=\Theta_{n m}, \hat{\theta}_{5}=$ $\theta_{5, n m}$ and $\hat{\theta}_{6}=\theta_{6, n m}$, respectively. In order to attain the convergence of task errors $e$ less or equal to $10^{-3}$ in approximately the same time, the following numerical values of gain coefficients for both controllers are taken: $K=195, \alpha=374.5, \lambda_{0}=1, \lambda_{1}=21, \lambda_{2}=16$, $\Lambda=163, \kappa=6, \eta=0.05$ and $\beta=\frac{5}{7}$, respectively. Moreover, due to the form of $D$, mappings $\alpha_{0}$ and $\alpha_{1}$ may be estimated as $\alpha_{0}=2\|q-q(0)\|+2\left\|y_{1}\right\|$, $\alpha_{1}=2\left\|y_{1}\right\|+2\left\|y_{2}\right\|$. Observe that filtered tracking error $r$ in (42) plays a role of linear sliding variable in the term $Y \hat{\Theta}+K r-J^{T} e$ which corresponds to $u_{n}$.
Furthermore, task error $e$ comes also linearly in control law (42). Additionally, controller (42) from [38, 39 ] requires the accurate measurement of $\dot{q}$. In addition, application of control algorithm (42) requires the full knowledge of the set $X$, i.e., we assume that $X=X_{n m}$. Under such conditions, control law (42) from $[38,39]$ is shown to be asymptotically stable. In order to better visualise time courses of the task errors for both controllers, we omit in the simulations an initial approaching phase of the end-effector to desired trajectory $p_{d}$. Consequently, the trajectory tracking is

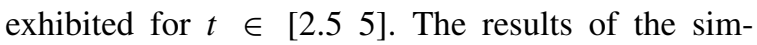
ulations are depicted in Figs. 2-9. As is seen from
Fig. 11 Task error $e_{2}$ for controller (41) with both linearly and non-linearly parametrizable discontinuous friction terms

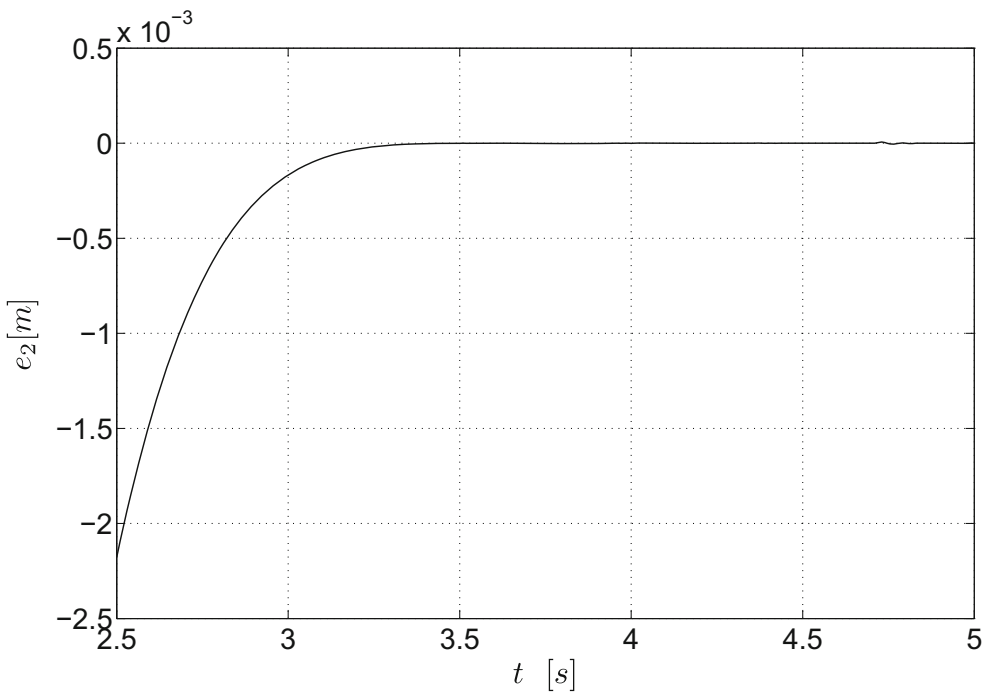


Fig. 12 Torque $v_{c, 1}$ for controller (41) with both linearly and non-linearly parametrizable discontinuous friction terms

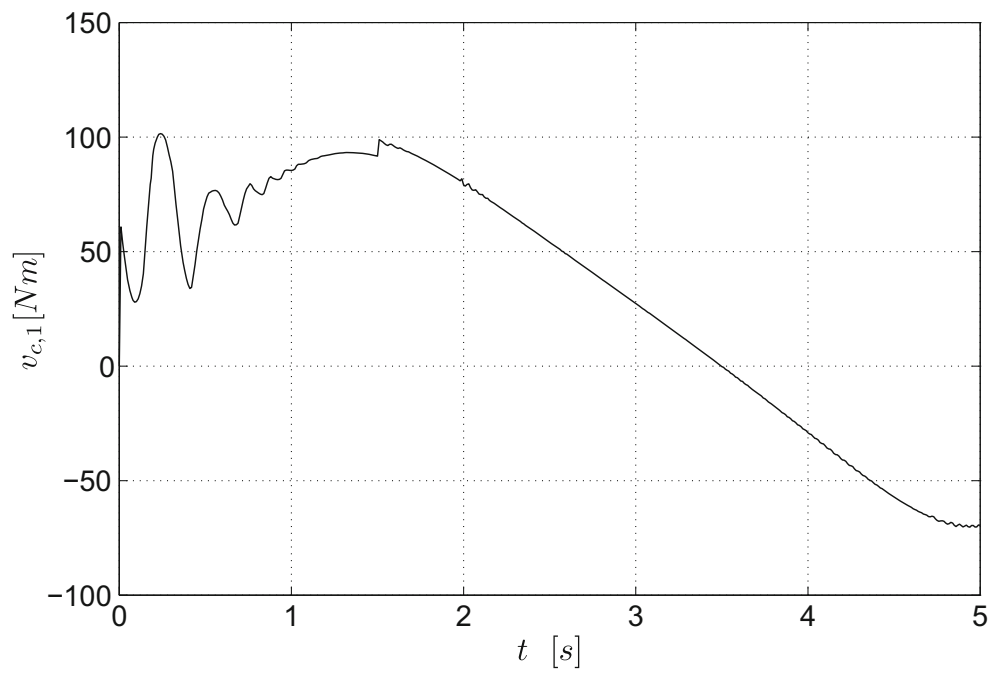

Figs. 2-3, 6-7 our controller generates tracking errors $e$,which are practically equal to zero for $T \geq 3.0$ whereas $e \neq 0$ for control law (42). This fact is a consequence of Remark 5 and the linear dependence of $Y \hat{\Theta}+K r-J^{T} e$ on $r$ and $e$. As is also seen from Figs. 4-5, 8-9, control scheme (41) provides smaller control signals (torques) in the sense of Chebyshev's norm (the maximum norm in $L^{\infty}$ ) than that given by Eq. 42. Let us note that the right-hand side of controller (28) or Eq. 41 presents a locally bounded Lebesgue measurable mapping. Numerical integration of such a class of functions using e.g. standard RungeKutta solvers of the 45 order requires a small value of integration step to obtain finite-time stability of our control law. In practice, the integration step should take the values less than or equal to $10^{-5}-10^{-4}$. The numerical simulations carried out with integration step equal to $10^{-3}$ has led to unstable work of controller (28). Moreover, due to finite integration step, which corresponds to finite sampling frequency in experiments, by solving differential (26)-(28) and related with it a numerical drift, task error $e$ does not equal (theoretically) zero for $t \geq T$ but takes very small values (less than or equal to $10^{-8}$ ). Then, both linearly and non-linearly parametrizable discontinuous friction terms of the form $5 \operatorname{sign}(\dot{q})+$ $2 \exp \left(-0.2\|\dot{q}\|^{2}\right) \operatorname{sign}(\dot{q})$, exhibiting the Coulomb and Stribeck effects [48, 69] have been added to disturbed manipulator dynamic equations considered in the previous experiment. The same controller
Fig. 13 Torque $v_{c, 2}$ for controller (41) with both linearly and non-linearly parametrizable discontinuous friction terms

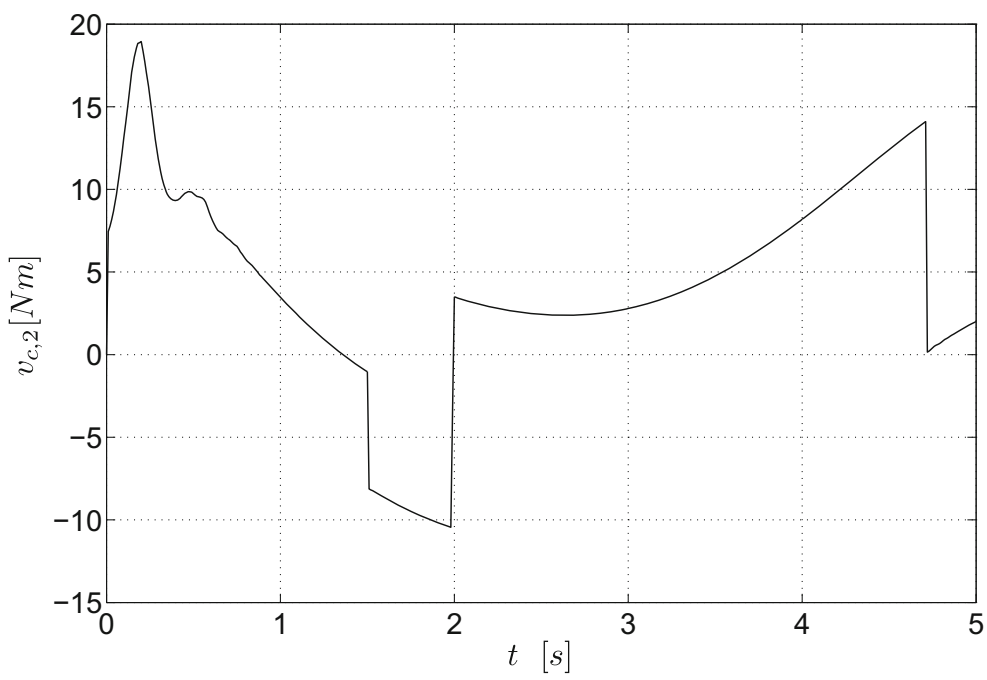


Fig. 14 Norm of task error $\|e\|$ for controller (41) with measurement noise

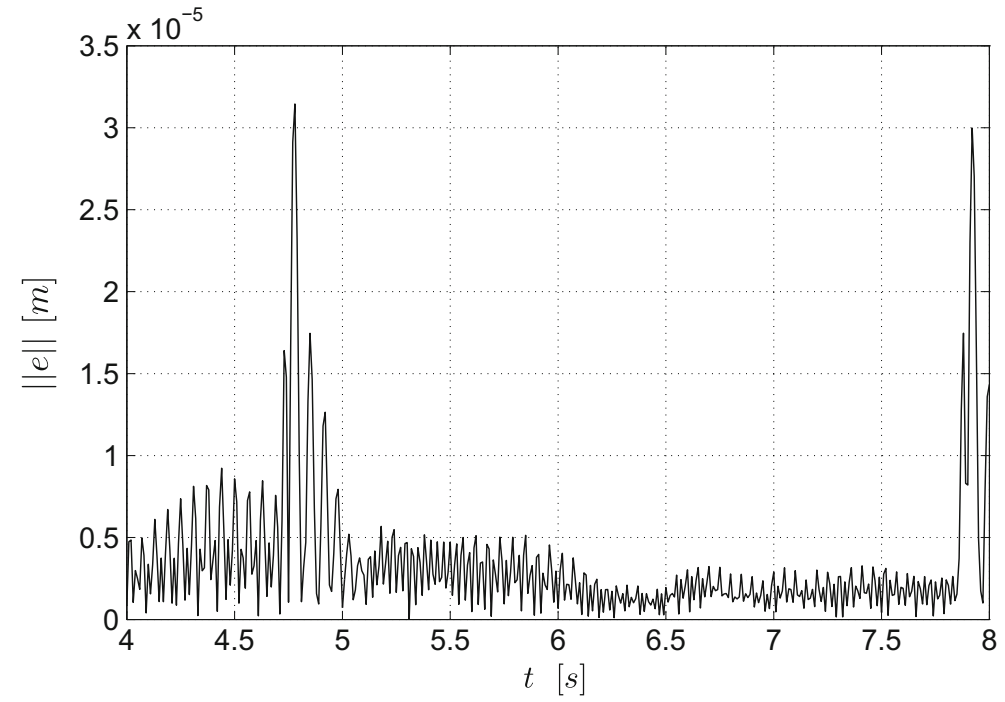

(41) as that from the first experiment with the same controller gains has been applied in the second experiment. In order to better visualise time courses of the task errors, we omit in the simulation an initial approaching phase of the end-effector to desired trajectory $p_{d}$. Consequently, the trajectory tracking for controller (41) is exhibited for $t \in$ $[2.5,5]$. The results of the simulations are depicted in Figs. 10-13 which show stable finite-time convergence of the end-effector to desired trajectory $p_{d}$. As is seen from Figs. 10-11, the control performance does not degrade even at the time instances $\{1.5,1.9,4.8\}$ corresponding to discontinuity of the term $5 \operatorname{sign}(\dot{q})+2 \exp \left(-0.2\|\dot{q}\|^{2}\right) \operatorname{sign}(\dot{q})$ which results in sudden changes of $v_{c}$ in Figs. 12-13. Nevertheless, transient variations of $v$ in a neighbourhood of the set of time moments $\{1.5,1.94 .8\}$ still present absolutely continuous mappings as it follows from Eq. 41, (39)-(40), (28). The torques produced by the controller (41) take approximately the values greater than $50 \mathrm{Nm}$ and less than $100 \mathrm{Nm}$ (see Figs 4, 12, $5,13)$. Let us note that the first coordinate of gravity term $G$ of EDDA takes the value of the order of $100 \mathrm{Nm}$ for $q_{1} \simeq 0$. As is seen from Fig. 1, accurate tracking of the desired trajectory $p_{d}$ forces coordinate $q_{1}$ to take the value $q_{1}=0$ at some time instant. Consequently, in order to compensate for gravity force at $q_{1}=0$, controller (41) should at least generate
Fig. 15 Joint velocity $\dot{q}_{1}$ for controller (41) with measurement noise

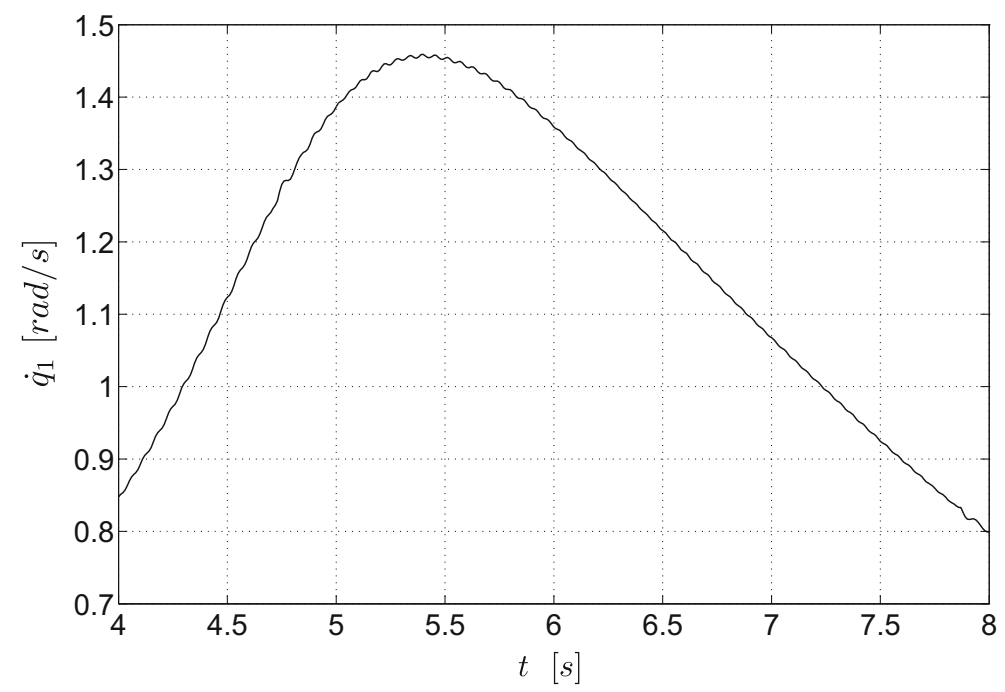


Fig. 16 Joint velocity $\dot{q}_{2}$ for controller (41) with measurement noise

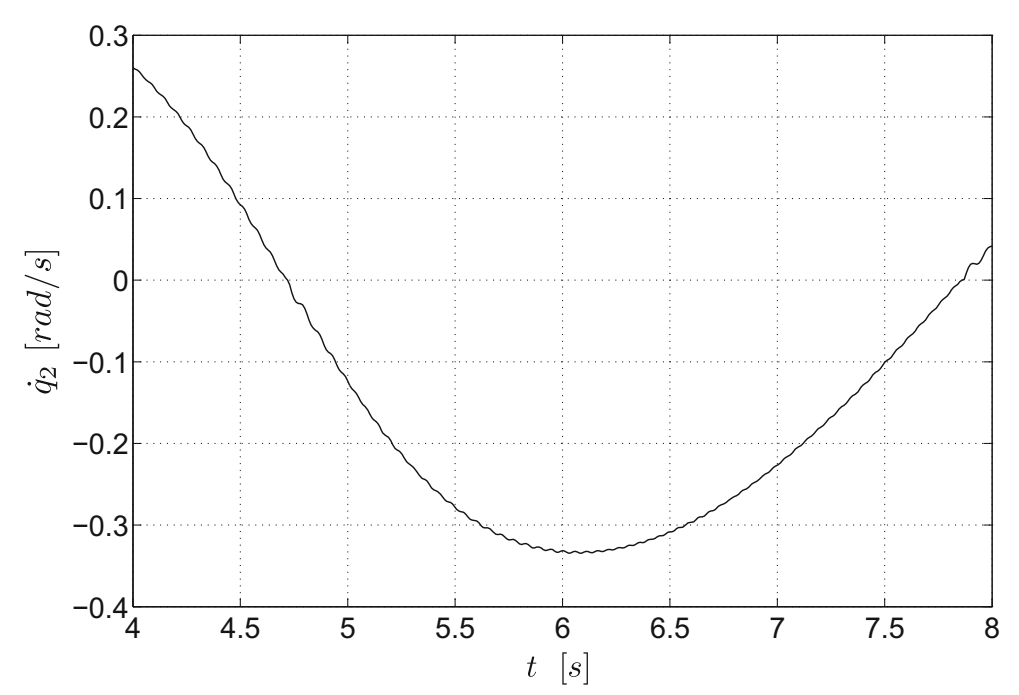

torque values greater than or equal to $100 \mathrm{Nm}$. We have additionally carried out the third numerical simulation subject to a measurement noise. Namely, both measured joint position $q$ and task error $e$, obtained from encoders, have been additionally contaminated by a measurement noise $\zeta(t)$ of a Brownian motion of the form $d \zeta(t)=10^{-5} \sqrt{t} X(t) d t ; X(t) \sim N(0,1)$ for $t \in[0,8]$. In such case, we have tested our controller under conditions of the second experiment for trajectory $p_{d}$. In order to better visualise time courses of the task error, we omit in the simulation an initial approaching phase of the end-effector to desired trajectory $p_{d}$. Consequently, the trajectory tracking for controller (41) is exhibited for $t \in[4,8]$. The results of simulation are given in Figs. 14-16 which indicate a good performance of controller (39)-(40), (41) subject to measurement noise. Two peaks of $\|e\|$ in Fig. 14 for $t \in\{4.8,7.9\}$ are result of the Coulomb and Stribeck discontinuity friction terms caused by joint velocity $\dot{q}_{2}$ (see Figs. 15, 16).

\section{Conclusions}

A new class of robust task space absolutely continuous TSM controllers with the finite-time convergence property of the trajectory tracking by $n$-DoF rigid robotic manipulator has been proposed in this paper. Moreover, a novel TSM manifold, making it possible to simultaneously apply both the first and second order sliding mode control techniques with their advantages, was incorporated into control scheme. Although our dynamically computed torque technique needs knowledge about the system equations of the robot, the approach is able to handle uncertainty (in both kinematics and dynamics as well as disturbance) occurring in the system. It is worth to emphasise the fact that the proposed absolutely continuous (chattering-free) controllers, based on the estimation of the transposed Jacobian matrix, are able to cope with globally unbounded disturbances acting on the robotic manipulators as well as singular configurations which can potentially occur when tracking a desired trajectory by the end-effector.

Open Access This article is distributed under the terms of the Creative Commons Attribution 4.0 International License (http:// creativecommons.org/licenses/by/4.0/), which permits unrestricted use, distribution, and reproduction in any medium, provided you give appropriate credit to the original author(s) and the source, provide a link to the Creative Commons license, and indicate if changes were made.

\section{References}

1. Bartolini, G., Ferrara, A., Punta, E.: Multi-input secondorder sliding-mode hybrid control of constrained manipulators. Dyn. Control 10(3), 277-296 (2000)

2. Bartolini, G., Ferrara, A., Usai, E., Utkin, V.I.: On multiinput chattering-free second-order sliding mode control. IEEE Trans. Autom. Control 45(9), 1711-1717 (2000)

3. Bartolini, G., Pisano, A., Punta, E., Usai, E.: A survey of applications of second-order sliding mode control to mechanical systems. Int. J. Control 76(9-10), 875-892 (2003) 
4. Feng, Y., Yu, X., Man, Z.: Non-singular terminal sliding mode control of rigid manipulators. Automatica 38(12), 2159-2167 (2002)

5. Ferrara, A., Capisani, L.M.: Second order sliding modes to control and supervise industrial robot manipulators. In: Fridman, L., et al (eds.) Sliding modes, LNCIS, Lecture Notes in Control and Information Sciences, vol. 412, pp. 541-567 (2011)

6. Filippov, A.F.: Differential Equations with Discontinuous Right-hand Side. Kluwer, Dordrecht (1988)

7. Fridman, L.: Singularly perturbed analysis of chattering in relay control systems. IEEE Trans. Autom. Control 47(12), 2079-2084 (2002)

8. Spong, M.W., Vidyasagar, M.: Robot dynamics and control. Wiley, New York (1989)

9. Levant, A.: Finite-time stability and high relative degrees in sliding-mode control. In: Fridman, L., et al (eds.) Sliding modes, LNCIS, Lecture Notes in Control and Information Sciences, vol. 412, pp. 59-92 (2011)

10. Levant, A.: Higher-order sliding modes, differentiation and output-feedback control. Int. J. Control 76(9-10), 924-941 (2003)

11. Levant, A., Livne, M.: Exact differentiation of signals with unbounded higher derivatives. IEEE Trans. Autom. Control 57(4), 1076-1080 (2012)

12. Yu, S., Yu, X., Shirinzadeh, B., Man, Z.: Continuous finitetime control for robotic manipulators with terminal sliding mode. Automatica 41, 1957-1964 (2005)

13. Zhao, D., Li, S., Gao, F.: A new terminal sliding mode control for robotic manipulators. Int. J. Control 82(10), 1804 1813 (2009)

14. Mondal, S., Mahanta, C.: Adaptive second order terminal sliding mode controller for robotic manipulators. J. Franklin Inst. 351, 2356-2377 (2014)

15. Galicki, M.: Finite-time control of robotic manipulators, Automatica 51, 49-54 (2015)

16. Khatib, O.: A unified approach for motion and force control of robot manipulators. IEEE J. Robot. Autom. RA-3(1), 43-53 (1987)

17. Hsu, P., Hauser, J., Sastry, S.: Dynamic control of redundant manipulators. J. Robot. Syst. 6, 133-148 (1989)

18. Canudas, C., Siciliano, B., Bastin, G. (eds.): Theory of Robot Control. Springer, London (1996)

19. Siciliano, B., Sciavicco, L., Villani, L., Oriolo, G. (eds.): Robotics. Modelling, Planning and Control. Springer Verlag (2010)

20. Galicki, M.: Path following by the end-effector of a redundant manipulator operating in a dynamic environment. IEEE Trans. Robot. 20(6), 1018-1025 (2004)

21. Kelly, R., Moreno, J.: Manipulator motion control in operational space using joint velocity inner loop. Automatica 41, 1423-1432 (2005)

22. Nakanishi, J., Cory, R., Mistry, M., Peters, J., Schaal, S.: Operational space control: A theoretical and empirical comparison. Int. J. Robot. Res. 27(6), $737-$ 757 (2008)

23. Moreno-Valenzuela, J., Gonzales-Hernandez, L.: Operational space trajectory tracking control of robot manipulators endowed with a primary controller of synthetic joint velocity. ISA Trans. 50, 131-140 (2011)
24. Pajak, G., Pajak, I.: Planning of an optimal collision-free trajectory subject to control constraints. In Proc. 2nd Int. Workshop on Robot Motion and Control, pp. 141-146 (2001)

25. Pajak, G., Pajak, I., Galicki, M.: Trajectory planning of multiple manipulators. In Proc. 4th Int. Workshop on Robot Motion and Control, pp. 121-126 (2004)

26. Tatlicioglu, E., Braganza, D., Burg, T.C., Dawson, D.M.: Adaptive control of redundant robot manipulators with subtask objectives. In: Proc. ACC, pp. 856-860 (2008)

27. Sadeghian, H., Keshmiri, M., Villani, L., Siciliano, B.: Priority oriented adaptive control of kinematically redundant manipulators. In: Proc. IEEE RA, pp. 293-298 (2012)

28. Sadeghian, H., Villani, L., Kesmiri, M., Siciliano, B.: Dynamic multi-priority control in redundant robotic systems. Robotica 31, 1155-1167 (2013)

29. Feng, G., Palaniswami, M.: Adaptive control of robot manipulators in task space. IEEE Trans. Automat. Contr. 38(1), 100-104 (1993)

30. Zergeroglu, E., Dawson, D.M., Walker, I., Behal, A.: Nonlinear tracking control of kinematically redundant robot manipulators (2000)

31. Braganza, D., Dixon, W.E., Dawson, D.M., Xian, B.: Tracking control for robot manipulators with kinematic and dynamic uncertainty. In: Proc. CDC, pp. 5293$5297(2005)$

32. Braganza, D., Dixon, W.E., Dawson, D.M., Xian, B.: Tracking control for robot manipulators with kinematic and dynamic uncertainty. Int. J. Robot. Autom. 23(2), 117-126 (2008)

33. Galicki, M.: Adaptive path-constrained control of a robotic manipulator in a task space. Robotica 25(1), 103-112 (2007)

34. Cheah, C.C., Liu, C., Slotine, J.J.: Adaptive tracking control for robots with unknown kinematic and dynamic properties. Int. J. Robot. Res. 25(30), 283-296 (2006)

35. Li, X., Cheah, C.C.: Adaptive regional feedback control of robotic manipulator with uncertain kinematics and depth information. In: Proc. ACC, pp. 5472-5477 (2012)

36. Li, X., Cheah, C.C.: Global task-space adaptive control of robot. Automatica 49, 58-69 (2013)

37. Galicki, M.: Inverse-free control of a robotic manipulator in a task space. Robot. Autonom. Syst. (2013). doi:10.1016/j.robot.2013.11.005

38. Zergeroglu, E., Sahin, H.T., Ozbay, U., Tektas, H.A.: Robust tracking control of kinematically redundant robot manipulators subject to multiple self-motion criteria. In: Proc. IEEE Control Appl.,pp. 2860-2865 (2006)

39. Ozbay, U., Sahin, H.T., Zergeroglu, E.: Robust tracking control of kinematically redundant robot manipulators subject to multiple self-motion criteria. Robotica 26, 711-728 (2008)

40. Singh, H.P., Sukavanam, N.: Neural network based control scheme for redundant robot manipulators subject to multiple self-motion criteria. Math. Comput. Model. 55, 12751300 (2012)

41. Nakamura, Y., Hanafusa, H.: Inverse kinematic solutions with singularity robustness for robot manipulator control. J. Dyn. Syst. Measur. Control 108, 163-171 (1986)

42. Wampler, C.W., Leifer, L.J.: Applications of damped leastsquares methods to resolved-rate and resolved-acceleration 
control of manipulators. J. Dyn. Syst. Measur. Control 110, 31-38 (1988)

43. Seraji, H., Colbaugh, R.: Improved configuration control for redundant robots. J. Robot. Syst. 6, 897-928 (1990)

44. Peng, Z.X., Adachi, N.: Compliant motion control of kinematically redundant manipulators. IEEE Trans. Robot. Automat. 9(6), 831-837 (1993)

45. Ott, C., Dietrich, A., Schaffer, A.A.: Prioritized multi-task compliance control of redundant manipulators. Automatica 53, 416-423 (2015)

46. Oh, Y., Chung, W.K.: Disturbance observer based motion control of redundant manipulators using inertially decoupled dynamics. IEEE/ASME Trans. Mechatronics 4(2), 133-146 (1999)

47. Colbaugh, R., Glass, K.: Robust adaptive control of redundant manipulators. J. Intell. Robot. Syst. 14, 69-88 (1995)

48. Haessing, D., Friedland, B.: On the modeling and simulation of friction. Trans. ASME J. Dyn. Syst. Measur. Control 113(3), 354-362 (1991)

49. Yoshikawa, T.: Manipulability of robotic mechanisms. Int. J. Robot. Res. 4(2), 3-9 (1985)

50. Maciejewski, A.A., Klein, C.A.: Obstacle avoidance for kinematically redundant manipulators in dynamically varying environments. I. J. Rob. Res. 4(3), 109-117 (1985)

51. Balleieul, J.: Kinematic programming alternatives for redundant manipulators (1985)

52. Wolovich, W.A., Elliot, H.: A computational technique for inverse kinematics. In: Proc. 23rd IEEE Conference on Decision and Control, pp. 1359-1363 (1984)

53. Siciliano, B.: A closed-loop inverse kinematic scheme for on-line joint-based robot control. Robotica 8, 231-243 (1990)

54. Kelly, R.: Robust asymptotically stable visual servoing of planar robots. IEEE trans. Rob. Automat. 12(5), 759-766 (1996)

55. Cheah, C.C.: On duality of inverse Jacobian and transpose Jacobian in task-space regulation of robots. In: Proc. IEEE Int. Conf. on robotics and automation, pp. 2571-2576 (2006)

56. Cheah, C.C., Lee, K., Kawamura, S., Arimoto, S.: Asymptotic stability control with approximate Jacobian matrix and its application to visual servoing. In: Proc. IEEE decision and control, pp. 3939-3944 (2000)

57. Moosavian, S.A.A., Papadopoulos, E.: Modified transpose Jacobian control of robotic systems. Automatica, 12261233 (2007)

58. Berghuis, H., Nijmeier, H.: Global regulation of Robots using only Position Measurements. Syst. Control Lett. 21(4), 289-293 (1993)

59. Vihonen, J., Honkakorpi, J., Mattila, J., Visa, A.: Geometryaided angular acceleration sensing of rigid multi-body manipulator using mems rate gyros and linear accelerometers. In: Proc. IEEE/RSJ IROS, pp. 2514-2520 (2013)

60. Canudas de Wit, C., Fixot, N., Astrom, K.J.: Trajectory tracking in robot manipulators via nonlinear estimated state feedback. IEEE Trans. Robot. Automat. 8(1), 138-144 (1992)
61. ElBeheiry, E.M., Zaki, A., ElMaraghy, W.H.: A unified approach for independent manipulator joint acceleration control and observation. ASME Dynamic Systems and Control Division 72(1), 659-666 (2003)

62. Khalil, H.K., Praly, L.: High-gain observers in nonlinear feedback control. Int. J. Robust and Nonlinear Control 24(6), 993-1015 (2014)

63. Ball, A.A., Khalil, H.K.: A nonlinear high-gain observer for systems with measurement noise. IEEE Trans. Automat. Control 58, 569-580 (2013)

64. De Luca, A., Schroder, D., Thummel, M.: An Accelerationbased State Observer for Robot Manipulators with Elastic Joints. In: Proc. IEEE international conference on robotics and automation, pp. 3817-3823 (2007)

65. Hsiao, T., Weng, M.C.: Robust joint position feedback control of robot manipulators. J. Dyn. Syst. Meas. Control. 135 (2013). doi:10.1115/1.4023669

66. Davila, J., Fridman, L., Levant, A.: Second-order sliding mode observer for mechanical systems. IEEE Trans. Automat. Control 50(11), 1785-1789 (2005)

67. Han, J.D., He, Y.Q., Xu, W.L.: Angular acceleration estimation and feedback control: An experimental investigation. Mechatronics 17, 524-532 (2007)

68. Atasi, A.N., Khalil, H.K.: Separation results for the stabilization of nonlinear systems using different high-gain observer designs. Syst. Control Lett. 39, 183-191 (2000)

69. Canudas de Wit, C., Ollson, H., Astrom, K., Lischinsky, P.: A new model for control of systems with friction. IEEE Trans. Automat. Contr. 40(3), 419-425 (1995)

70. Galicki, M.: Finite-time trajectory tracking control in a task space of robotic manipulators. Automatica 67, 165-170 (2016)

71. Hong, Y., Xu, Y., Huang, J.: Finite-time control for robot manipulators. Syst. Control Lett. 46, 243-253 (2002)

72. Fridman, L., Moreno, J., Iriarte, R. (eds.): Sliding modes after the first decade of the 21-st Century - State of the art. Springer (2011)

73. Tchon, K., Mazur, A., Duleba, I., Hossa, R., Muszynski, R.: Manipulators and mobile robots. Modelling, motion planning and control. Akademicka Oficyna Wydawnicza PLJ (in Polish) (2000)

Mirosław Galicki received the M. Sc. degree from the Technical University of Wroclaw, Poland, in 1980 and the Ph. D. degree in electrical and computer sciences from the Technical University of Wroclaw. He was with the Institute of Technical Cybernetics of Wroclaw from September 1981-1984. Since 1985 he has been at the University of Zielona Gora, Poland, and from 1992-2013 he was also with the Institute of Medical Statistics, Computer Sciences and Documentation of the Friedrich Schiller University Jena, Germany. His research interests include control of dynamic systems with both holonomic and non-holonomic constraints, structural optimization and optimal control problems. Areas of particular interest include the problems of motion control of both redundant stationary and mobile manipulators. 